Canadian Journal of Regional Science

Revue canadienne des sciences régionales

\title{
Caractéristiques locales et individuelles : une analyse spatiale de la croissance des établissements en région non métropolitaine
}

\section{Ismaëlh Ahmed Cissé et Jean Dubé}

Volume 43, numéro 1, 2020

Soumis : 28 mai 2019

Accepté : 8 juin 2020

URI : https://id.erudit.org/iderudit/1083580ar

DOI : https://doi.org/10.7202/1083580ar

Aller au sommaire du numéro

\section{Éditeur(s)}

Canadian Regional Science Association / Association canadienne des sciences régionales

\section{ISSN}

0705-4580 (imprimé)

1925-2218 (numérique)

Découvrir la revue

\section{Citer cet article}

Cissé, I. A. \& Dubé, J. (2020). Caractéristiques locales et individuelles : une analyse spatiale de la croissance des établissements en région non métropolitaine. Canadian Journal of Regional Science / Revue canadienne des sciences régionales, 43(1), 39-57. https://doi.org/10.7202/1083580ar

\section{Résumé de l'article}

L’objectif de cet article est de vérifier si les caractéristiques locales et/ou individuelles influencent la croissance de l'emploi des nouveaux établissements dans la région non métropolitaine du Bas-Saint-Laurent (BSL) au Québec. À partir des microdonnées de nouveaux établissements créés entre 2008 et 2014, des indicateurs de concentration spatiale caractérisent le milieu des établissements dans un rayon de 1250 mètres. Une modélisation en deux étapes par la procédure de Heckman (1979) a permis, d'une part, de modéliser la survie (modèle probit) des nouveaux établissements et, d'autre part, de corriger le biais de sélection et d'estimer la croissance de l'emploi des nouveaux établissements (modèle SDM-Spatial Durbin Model). Les résultats montrent que les caractéristiques individuelles et locales influencent la survie des établissements. Quant à la croissance de l'emploi des nouveaux établissements, c'est en période de récession que le milieu fait la différence. En plus d'être influencée par les caractéristiques individuelles, la spécialisation industrielle relative au secteur du commerce \& du transport et la variété reliée se présentent comme des milieux favorables à la croissance des établissements en période de récession. 


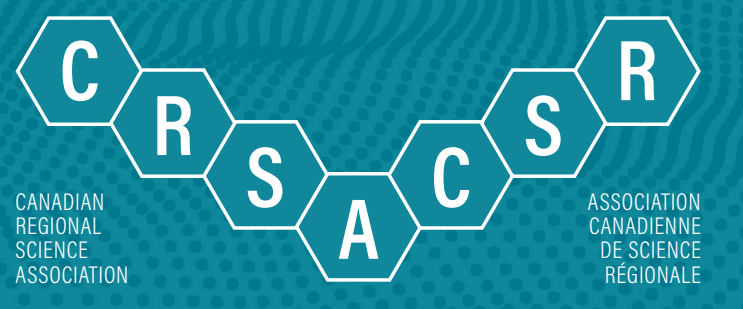

\section{CARACTÉRISTIQUES LOCALES ET INDIVIDUELLES: UNE ANALYSE SPATIALE DE LA CROISSANCE DES ÉTABLISSEMENTS EN RÉGION NON MÉTROPOLITAINE}

\section{Ismaëlh Ahmed Cissé, Jean Dubé}

\author{
Ismaëlh Ahmed Cissé \\ Étudiant au doctorat en ATDR \\ École supérieure d'aménagement du territoire \\ et de développement régional (ÉSAD) \\ Université Laval \\ 2325, rue des Bibliothèques \\ Québec, Canada, G1V 0A6 \\ ismaelh-ahmed.cisse.1@ulaval.ca
}

\author{
Jean Dubé \\ Professeur titulaire \\ École supérieure d'aménagement du territoire \\ et de développement régional (ÉSAD) \\ Université Laval \\ 2325, rue des Bibliothèques \\ Québec, Canada, G5L 3A1 \\ jean.dube@esad.ulaval.ca
}

Soumis: 28 mai 2019

Accepté : 8 juin 2020

Résumé: L'objectif de cet article est de vérifier si les caractéristiques locales et/ou individuelles influencent la croissance de l'emploi des nouveaux établissements dans la région non métropolitaine du Bas-Saint-Laurent (BSL) au Québec. À partir des microdonnées de nouveaux établissements créés entre 2008 et 2014, des indicateurs de concentration spatiale caractérisent le milieu des établissements dans un rayon de 1250 mètres. Une modélisation en deux étapes par la procédure de Heckman (1979) a permis, d'une part, de modéliser la survie (modèle probit) des nouveaux établissements et, d'autre part, de corriger le biais de sélection et d'estimer la croissance de l'emploi des nouveaux établissements (modèle SDM-Spatial Durbin Model). Les résultats montrent que les caractéristiques individuelles et locales influencent la survie des établissements. Quant à la croissance de l'emploi des nouveaux établissements, c'est en période de récession que le milieu fait la différence. En plus d'être influencée par les caractéristiques individuelles, la spécialisation industrielle relative au secteur du commerce \& du transport et la variété reliée se présentent comme des milieux favorables à la croissance des établissements en période de récession.

Mots clés: Modèle de croissance spatiale, croissance d'entreprise, proximité, région non métropolitaine.

JEL: C21, L25, R12, O18 
seconde section présente le cadre conceptuel sur lequel s'appuie

La performance d'une entreprise est généralement associée à son succès, qui peut se mesurer par la survie, la croissance (financière, part de marché, capital humain) et la rentabilité (Belleau-Arsenault \& Dubé, 2019; De Vaan et al., 2013; Kauppila, 2015; Kiviluoto, 2013; Richard et al., 2009). Parmi l'éventail d'indicateurs, Wall et al. (2004) suggèrent d'utiliser la mesure qui répond le mieux à l'objectif de la recherche. En science régionale, la performance individuelle des entreprises est l'un des facteurs fondamentaux qui influencent les trajectoires de développement économique régional et local. Pour les décideurs publics, il est important d'identifier le type d'entreprise qui crée de l'emploi et qui conséquemment favorise le développement local. L'emploi est un des indicateurs qui exprime la croissance des entreprises et, par conséquent, reflète sa performance.

Pour expliquer la croissance des entreprises, les caractéristiques individuelles telles que l'âge et le secteur d'activité sont souvent mises en avant (Giunta et al., 2012; Haltiwanger et al., 2013) tout comme les économies d'échelles qui seraient le fruit d'une concentration spatiale d'activités économiques. Bien que les économies d'agglomérations soient considérées comme surtout liées aux grandes villes, plusieurs études ont démontré qu'il existe, en régions éloignées et peu peuplées, une concentration naturelle d'activités économiques qui favorisent la performance des entreprises (Brunelle \& Dubé, 2018), mais également la décision de localisation des établissements (Cissé et al., 2020; Dubé et al., 2016). Les fondements des économies d'agglomération reposent sur des principes microéconomiques, ce qui nécessite la prise en compte simultanée des caractéristiques individuelles et locales (de Groot et al., 2016). Ainsi, les caractéristiques locales notamment les économies d'agglomérations peuvent aussi expliquer la croissance des entreprises (Rosenthal \& Strange, 2004).

L'objectif de cet article est de vérifier si, dans un contexte non métropolitain, la croissance de l'emploi des nouveaux établissements est liée aux caractéristiques locales, telles que la concentration d'établissements du même secteur ou de secteurs différents, aux caractéristiques individuelles des établissements, ou un mixte des deux. L'article cherche également à comprendre comment l'effet d'une récession économique peut influencer les déterminants de la performance individuelle des entreprises.

Pour ce faire, l'analyse propose d'utiliser des microdonnées portant sur les établissements créés entre 2008 et 2014 dans la région du Bas-Saint-Laurent (BSL) afin d'expliquer leur croissance. Un ensemble d'indicateurs locaux, définis dans un rayon de 1250 mètres, sont construits selon une approche fondée sur la distance (ou distance based measure - Dubé \& Brunelle, 2014). Une modélisation en deux étapes à la Heckman (1979) est utilisée afin d'identifier, d'une part, les facteurs de survie (modèle probit) des nouveaux établissements, et, d'autre part, corriger le biais de sélection (pour les entreprises qui survivent) pour modéliser le taux de croissance de l'emploi, en fonction de leurs caractéristiques individuelles, mais aussi en fonction de celles des établissements autour à partir d'un modèle de type Durbin spatial (SDM - Spatial Durbin Model).

Pour ce qui est de la survie, les résultats montrent que les caractéristiques individuelles et locales influencent la survie des établissements. Quant à la croissance de l'emploi des nouveaux établissements, elle varie selon le secteur. À l'exception des effets d'urbanisation, les caractéristiques locales jouent un rôle plus important dans la croissance de l'emploi des nouveaux établissements en période de récession. Par contre, les caractéristiques individuelles influencent la croissance de l'emploi des nouveaux des établissements, quelle que soit la conjoncture économique.

L'article s'articule autour de six sections. La première section présente une revue de littérature sur la croissance des entreprises. La la méthodologie. Le modèle économétrique est ensuite développé pour adapter le cadre conceptuel au contexte d'étude. Une troisième section est dédiée à la présentation des données utilisées, elle est suivie de la section des résultats. Une discussion autour ces résultats est élaborée dans une cinquième section et enfin la section de la conclusion pour clôturer l'article.

\section{REVUE DE LITTÉRATURE}

\section{Les indicateurs de la croissance}

Les indicateurs de la croissance d'une entreprise peuvent être regroupés en trois catégories, qu'ils soient exprimés de façon absolue -nombre, ou relative -pourcentage. II s'agit entre autres i) des intrants: fonds d'investissement, capital et nombre employés; ii) des extrants: chiffre d'affaires, bénéfices et productivités; et de iii) la valeur de l'entreprise: actifs, valeur ajoutée économique, capitalisation boursière (Bonča et al., 2018; Daunfeldt et al., 2014; Frédéric Delmar et al., 2003; Garnsey et al., 2006).

L'emploi est un indicateur qui représente une mesure comparable décrivant le processus interne d'organisation, d'adaptation de l'entreprise face aux changements sectoriels, le niveau et l'orientation de l'expansion d'une entreprise (Tang, 2015). Les changements du niveau d'emploi permettent de mesurer la stabilité de la croissance contrairement au chiffre d'affaires ou au capital, qui peuvent être très volatiles (Garnsey et al., 2006). Dans une perspective de développement régional, l'emploi est un indicateur qui permet d'inférer sur le degré d'insertion sociale et de cohésion des communautés (Brunelle \& Shearmur, 2007). De plus, l'emploi peut apporter de l'information sur l'activité industrielle et le capital humain local.

La croissance peut être exprimée de manière organique (interne) ou refléter une fusion-acquisition d'entreprises (Daunfeldt et al., 2014). Lorsque la croissance est mesurée par le niveau d'emploi, les données des établissements reflètent plus fidèlement une croissance organique, car elle n'est pas associée à des fusions ou acquisitions (Dixon \& Rollin, 2012).

Même si le nombre d'employés ne reflète pas de manière parfaite les variations de la productivité du travail, la substitution des machines aux hommes, le degré d'intégration et les autres décisions d'achat ou de vente (Delmar et al., 2003), l'emploi est un indicateur qui a l'avantage de ne pas être sensible à l'inflation ou au taux de change. II s'avère être un indicateur de choix dans une analyse multisectorielle (Coad, 2009).

\section{Les déterminants de croissance des entreprises}

Pour expliquer la croissance des entreprises, deux facteurs sont généralement pris en compte (voir Zhou et al., 2018) : i) les facteurs internes propres à l'entrepreneur et aux caractéristiques individuelles des entreprises. ii) Les facteurs externes à l'entreprise, tels que les institutions (la législation, les impôts et les subventions gouvernementales), l'accès au financement, la dynamique économique locale et la conjoncture économique internationale.

Les facteurs internes propres à l'entrepreneur concernent les traits de personnalité du dirigeant, sa motivation, ses compétences -son niveau d'étude, son expérience professionnelle- et ses caractéristiques personnelles - son genre, son âge - (Baum et al., 2001; Burke et al., 2018; Cliff, 1998; Delmar, 1996; Nicholson, 1998).

Quant aux caractéristiques individuelles des entreprises, plusieurs études ont démontré qu'il existe une relation inverse entre le taux de croissance et la taille ou l'âge des entreprises (voir Giunta et al., 2012). Les petites entreprises par exemple grandissent plus rapi- 
dement afin d'atteindre la taille minimale efficace (Audretsch et al., 2004). Or, selon la Loi de Gibrat, le taux de croissance de l'emploi et la taille de l'entreprise sont indépendants. Pour les défenseurs de cette théorie, les entreprises «subissent des chocs idiosyncratiques comparables dont l'incidence et la magnitude sont indépendantes de la taille de l'entreprise. Le taux de croissance des entreprises est une conséquence de ces chocs» (Dixon \& Rollin, 2012, p. 11), et ce peu importe leur taille. C'est d'ailleurs pour son caractère idiosyncratique et la prédominance d'élément stochastique «hasard q que plusieurs études ont conclu de la difficulté de déterminer la trajectoire de croissance d'une entreprise (Coad, 2007; Macpherson \& Holt, 2007).

Pour mieux comprendre cette relation, Haltiwanger et al. (2013) expliquent que les chocs subits par les entreprises sont transitoires, ce qui les amène à revenir à leur moyenne de long terme, et crée plutôt une relation entre la taille et l'âge. Au Canada, Dixon \& Rollin (2012) ont montré qu'en contrôlant pour l'âge des entreprises, la croissance ne varie pas beaucoup en fonction de la taille. Toutefois, la croissance des plus petites entreprises évolue différemment des autres à cause des spécificités industrielles, de la conjoncture économique ou de l'âge des entreprises. Ces résultats corroborent ceux de Jovanovic (1982) selon lesquels la croissance des petites et/ou jeunes entreprises est systématiquement plus grande chez les plus efficientes. Au niveau des facteurs internes, il existe d'autres caractéristiques individuelles telles que: le secteur d'activité, la stratégie, le mode d'organisation, les perspectives d'orientation du marché, la capacité d'innover et le capital humain qui influencent la croissance des entreprises (Acosta et al., 2018; Goedhuys \& Sleuwaegen, 2016; Gupta et al., 2013; Wiklund et al., 2009).

Les facteurs externes jouent un rôle important dans la croissance des entreprises et peuvent, dans certains cas, représenter une barrière à la croissance pour les petites et moyennes entreprises. Ces barrières dépendent souvent de la localisation -rural ou urbain par exemple- et se présentent sous forme de barrières institutionnelles à travers le manque de soutien du gouvernement, le manque de maind'œuvre locale qualifiée, ou sous forme de barrières financières principalement les contraintes et difficultés à l'emprunt (Drinkwater et al., 2018; Lyee \& Cowling, 2015). Il en est de même de la conjoncture économique telle que la grande récession de 2008 et de son impact sur les entreprises. En effet, du fait de la crise, l'augmentation du taux de chômage dans les pays à une conséquence sur la consommation des ménages. De plus, l'augmentation de la dette publique impacte les services offerts par l'état et sa capacité à faire face aux problèmes politique et économique. Enfin, la difficulté des banques a prêter aux entreprises. Autant de facteurs qui ralentissent la croissance des entreprises et qui peuvent compromettre leurs survies en période de récession (Borra \& Gómez-García, 2016; Peric \& Vitezic, 2016; Cowling et al., 2015).

La théorie des économies d'agglomération (spécialisation, diversification et concurrence), en tant que facteur externe, met l'accent sur le rôle du positionnement géographique et lie la croissance, la productivité et la résilience des entreprises aux caractéristiques locales (Cainelli et al., 2016; Mameli et al., 2014; Rosenthal \& Strange, 2004). De façon empirique, Audretsch \& Dohse (2007) démontrent que la performance des entreprises de haute technologie, exprimée par la croissance de l'emploi, est positivement influencée par les caractéristiques locales et sectorielles des entreprises. Cainelli et al. (2016) montrent que la spécialisation a plus d'impact que la diversification sur la productivité totale des facteurs des entreprises manufacturières en Italie. En Angleterre, les effets de concentration industrielle (cluster) expliquent aussi bien l'augmentation de l'emploi des entreprises manufacturières (Beaudry \& Swann, 2009), que celle des services informatiques et de la recherche et développement (Fingleton et al., 2004).
II faut mentionner que les économies d'agglomérations sont fondamentalement d'ordre microéconomique, de ce fait les niveaux micro et macro doivent être modélisés simultanément (de Groot et al., 2016; van Oort et al., 2012). Ainsi, les caractéristiques individuelles et locales doivent être observées simultanément dans l'étude de la croissance des entreprises tout en considérant l'hétérogénéité des entreprises (petites/grandes, jeunes/anciennes, très productives/ moins productives) dans une même localité (Cainelli \& Ganau, 2018; Wang, 2015). En période de crise -facteur externe à l'entreprise-, en considérant l'âge (caractéristiques individuelles), les jeunes entreprises sont les plus impactées négativement (Criscuolo et al., 2014; Fort et al., 2013). Alors que la propension à l'exportation, la structure géographique des exportations, la propriété de l'entreprise (étrangère ou locale), l'indépendance aux sources de financement externe assurent une certaine résilience aux entreprises en période de crise (voir Burger et al. 2017). Au niveau empirique, Garsaa \& Levratto (2017) montrent qu'en France, les caractéristiques individuelles expliquent en majeure partie la croissance du nombre d'employés des entreprises alors que les caractéristiques locales l'expliquent marginalement. Par contre, Manzocchi et al. (2017) étudient la productivité totale des facteurs et trouvent que les caractéristiques locales expliquent plus la disparité de productivités entre des entreprises manufacturières en Italie. Selon Hoogstra \& van Dijk (2004), la localisation compte dans l'explication de la croissance des entreprises, mais l'effet diffère selon le secteur d'activité. Au Canada, Baldwin et al.(2010) ont montré que les économies d'agglomération contribuent plus à la productivité des entreprises manufacturières du même sous-secteur. Cependant, ce sont plutôt les grandes entreprises manufacturières à établissements multiples, plus anciennes et sous contrôle étranger, qui profitent davantage de la concentration spatiale des entreprises contrairement aux jeunes et petites entreprises canadiennes (Brown \& Rigby, 2013).

Bien que les effets des économies d'agglomérations soient beaucoup plus perceptibles dans les grandes villes, qui attirent les entreprises, certains ménages préfèrent s'éloigner des centres urbains (Partridge et al., 2010). Il peut donc exister un effet d'inversion lié notamment à la montée des externalités négatives, qui entraîne une migration de certaines activités économiques des grandes villes vers des régions éloignées et moins peuplées. L'effet d'inversion peut également s'expliquer par: i) une restructuration régionale, c'est-à-dire le passage d'une économie traditionnelle lourde à une économie de services (Frey \& Johnson, 1996), entraînant un déplacement des activités extractives et manufacturières vers les régions (Fuguitt \& Beale, 1996), et ii) une déconcentration, c'est-à-dire la migration des individus (par exemple des retraités) et des entreprises (gentrification rurale) vers des régions moins densément peuplées possédant de fortes aménités (Frey \& Johnson, 1996; Nelson \& Nelson, 2011).

En somme, un ensemble de facteurs peuvent expliquer des mouvements migratoires vers une région éloignée. Par contre, c'est seulement à partir d'un certain seuil de concentration que les économies d'agglomération peuvent expliquer les effets de localisation en région moins densément peuplée (Rupasingha et al., 2015). Au Québec, Cissé et al. (2020) ont montré que les caractéristiques locales à travers la spécialisation d'une petite ville comparée à une zone rurale favorisent la création d'entreprises dans le secteur manufacturier. Quant aux services, c'est plutôt la distance aux centres urbains qui influence la localisation des nouvelles entreprises.

Ce tour d'horizon de la littérature, conduit à poser les hypothèses suivantes pour la suite de l'analyse:

H1: L'âge des établissements est la principale caractéristique individuelle qui favorise la croissance de l'emploi des nouveaux établissements. 
H2: La croissance de l'emploi des nouveaux établissements diffère d'un secteur à un autre.

H3: La croissance de l'emploi des nouveaux établissements est sensible à la conjoncture économique.

H4: La proximité du marché influence la croissance de l'emploi des nouveaux établissements.

\section{CADRE CONCEPTUEL ET MÉTHODOLOGIE}

\section{Cadre conceptuel}

La méthodologie employée est principalement basée sur le modèle de croissance spatial proposé par Lesage \& Fischer (2008). Ce modèle économétrique, de type Durbin spatial (SDM — Spatial Durbin Model), tient non seulement compte des caractéristiques individuelles des établissements, mais également des caractéristiques des établissements voisins, de la structure de la connectivité spatiale entre les établissements, en plus d'inclure des effets de débordements spatiaux (locaux et globaux) pour expliquer la croissance régionale.

Le choix d'un modèle SDM pour expliquer la croissance est motivé par trois raisons. Premièrement, et d'un point de vue technique, la littérature sur les modèles de croissance montre qu'il existe une dépendance spatiale dans les résidus des modèles de régression linéaire estimés par les moindres carrés ordinaires (MCO) ou les moindres carrés généralisés (MCG), ce qui invalide les conclusions que l'on peut tirer de ces modèles. Deuxièmement, le modèle SDM permet de contrôler pour l'omission de variables spatialement dépendantes et corrélées avec certaines variables existantes dans le modèle. Par exemple, dans le modèle de croissance du revenu régional, le niveau initial du revenu régional est considéré comme une variable indépendante. Pourtant, le niveau initial du revenu régional est spatialement dépendant des autres variables indépendantes utilisées dans ces modèles (exemple: le revenu par habitant, l'emploi, la population, etc..). L'omission de variables du fait du manque de données sur des facteurs importants (le revenu par habitant par exemple) peut introduire des biais puisque certaines interactions spatiales ne sont considérées dans le modèle. Finalement, un autre avantage du modèle de type SDM est qu'il n'impose aucune restriction quant à l'ordre de grandeur des effets de débordement; il peut être différent pour chacune des variables explicatives (Elhorst, 2010). Mieux encore, il produit des estimations de coefficients non biaisées même si le véritable processus de génération de données est un SAR ou un SEM² (Piacentino et al., 2017).

À partir d'une réécriture du modèle de régression non spatial du taux de croissance de l'emploi pour une observation i entre le temps t et le temps 0 (équation 1), on obtient un modèle de croissance (équation 2) que l'on peut généraliser en y en incluant des décalages spatiaux pour la variable dépendante et les variables indépendantes afin de tenir compte des interactions spatiales possibles (équations 3 et 4).

Où $e_{i t}$ est le nombre d'employés à la date $t, e_{i 0}$ est le nombre d'emplois à la période initiale (création), qui diffère d'une observation à l'autre, et T représente l'âge de l'établissement à la date $t$,

Ce type de modèle suppose que le taux de croissance présente une dépendance spatiale liée à l'omission de variables explicatives. Les variables indépendantes sont exprimées à la période initiale afin d'éviter un problème de simultanéité. La période initiale est ainsi considérée comme une dotation pour expliquer la croissance future.

$$
\Delta e_{i t}=\left[\ln \left(e_{i t}\right)-\ln \left(e_{i 0}\right)\right] / T
$$

$$
\Delta e_{i t}=\phi \ln \left(e_{i 0}\right)+Z_{i 0} \theta+\varepsilon_{i t}
$$

$$
\left(I_{n}-\rho \mathrm{W}\right) \Delta e_{i t}=\phi_{1} \ln \left(e_{i 0}\right)+\phi_{2} \mathrm{~W} \ln \left(e_{i 0}\right)+\mathrm{Z}_{i 0} \theta_{1}+\mathrm{WZ} \mathrm{Z}_{i 0} \theta_{2}+\varepsilon_{i t}
$$

$$
\Delta e_{i t}=\rho \mathrm{W} \Delta e_{i t}+\phi_{1} \ln \left(e_{i 0}\right)+\phi_{2} \mathrm{~W} \ln \left(e_{i 0}\right)+\mathrm{Z}_{i 0} \theta_{1}+\mathrm{WZ} \mathrm{Z}_{i 0} \theta_{2}+\varepsilon_{i t}
$$

En simplifiant, le modèle de croissance spatial SDM peut s'écrire de manière plus compacte (équation 5).

$$
\Delta e_{i t}=\rho \mathrm{W} \Delta e_{i t}+\mathrm{X} \beta_{1}+\mathrm{WX} \beta_{2}+\varepsilon_{t}
$$

Où la matrice $X$ est composée du vecteur du phénomène étudié à la période initiale $\ln \left(e_{i 0}\right)$ et d'autres variables indépendantes $Z_{i 0}$ à la période initiale et $W$ est une matrice de pondérations spatiales. Le paramètre $\rho$ (scalaire) permet d'exprimer la dépendance spatiale de la variable dépendante, les vecteurs de paramètres $\beta_{1}$ et $\beta_{2}$, formés par les vecteurs de paramètres $\phi_{1}$ et $\theta_{1}$ et $\phi_{2}$ et $\theta_{2}$, respectivement sont à estimer, alors que $\varepsilon_{t}$ représente le vecteur de terme d'erreur.

\section{Modèle économétrique}

Le modèle de Lesage \& Fischer (2008) peut être transposé à une échelle micro - la croissance des établissements. La croissance individuelle d'un établissement est alors liée non seulement de ses propres caractéristiques, mais aussi à son environnement immédiat du fait des effets de débordement. La matrice $X$ de l'équation 5 est aussi composée de variables de caractéristiques locales, qui peuvent être construites à partir de la méthode basée sur la distance (Distance-Based Measures - DBM). ${ }^{3}$ Ces variables mesurent les effets de concentration ou de dispersion géographique des entreprises dans un rayon donné.

L'étude de la croissance des établissements est généralement basée sur ceux qui sont encore en activité, et qui ont survécu durant la période d'étude. Ce choix méthodologique a pour effet de limiter les observations de croissance négative, car les établissements qui n'ont pas survécu ont nécessairement enregistré une croissance négative (ou au mieux nulle si l'établissement n'avait pas d'employés). En opposition, l'application du modèle sans corriger pour le biais d'attrition a pour effet de maximiser la présence d'établissements avec des caractéristiques particulières (elles ont survécu) (voir Hoogstra \& van Dijk, 2004). Ce problème a pour effet d'entraîner un biais de sélection de l'échantillon, et ainsi invalider les résultats puisque les inférences statistiques sont basées sur un échantillon non aléatoire et non représentatif.

Pour corriger le potentiel biais d'attrition (ou de sélection), une modélisation en deux étapes par la procédure de Heckman (1979) est utilisée. La première étape consiste à estimer un modèle de sélection, de type probit ou logit, afin de déterminer la probabilité de survie d'un établissement en fonction de ses caractéristiques individuelles et locales ${ }^{4}$ (équation 6).

$$
\operatorname{Pr}\left(S_{i t}=1\right)=\theta_{0}+X \theta_{2}+\varepsilon_{t}
$$

\footnotetext{
1 Spatial Autoregressive Model

2 Spatial Error Model

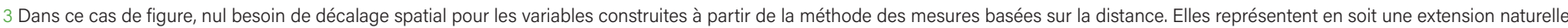
de l'expression WX.

4 Cas des variables construites à partir de la méthode des mesures basées sur la distance.
} 
Où la variable dépendante $S_{i t}$ prend la valeur 1 si l'établissement est toujours en activité à la date $t$ et 0 autrement. La matrice $X$ est la même que celle de l'équation 5. Comme le soulignent Serrasqueiro et al.(2010), cette approche suppose que les facteurs de croissance et de survie sont les mêmes.

L'estimation du modèle et des paramètres permet ainsi de reconstruire l'inverse du ratio de Mills, soit le rapport entre la densité de probabilité et la fonction de distribution cumulative pour chaque établissement (voir Heckman, 1979). Cette nouvelle variable peut alors être utilisée comme variable indépendante additionnelle dans la deuxième étape (équation 7) qui consiste à estimer un modèle SDM uniquement avec les établissements qui ont survécu afin de corriger le biais de sélection (Diao, 2015).

$$
\Delta e_{i t}=\rho \mathrm{W} \Delta e_{i t}+\mathrm{X} \beta_{1}+\mathrm{WX} \beta_{2}+I M R_{t} \beta_{3}+\varepsilon_{i t}
$$

Selon LeSage \& Pace (2014), l'une des spécificités du modèle SDM est l'impossibilité d'interpréter les paramètres $\beta_{1}$ et $\beta_{2}$ comme dans un modèle de régression linéaire à cause de la relation non linéaire qui existe entre la variable dépendante $\left(\Delta e_{t}\right)$ et les variables indépendantes de la matrice $X^{5}$ La dérivée partielle de l'une des variables explicatives $\left(X_{k}\right)$ de ce type de modèles est:

$$
\partial \Delta e / \partial X_{k}=\left(l_{n}-\rho W\right)^{-1}\left(l_{n} \beta_{k 1}+W \beta_{k 2}\right)=S_{k}(W)
$$

LeSage \& Pace (2009) proposent d'utiliser la moyenne de la diagonale de la matrice $S_{k}(W)$ pour calculer l'effet direct. Pour un établissement $i$, l'effet direct mesure l'impact moyen d'une variation de $X_{k}$ de l'établissement $i$ sur le taux de croissance de ce même établissement. L'effet indirect, qui est la moyenne des éléments hors de la diagonale de $S_{k}(W)$, mesure l'impact moyen d'une variation de $X_{k}$ des voisins de l'établissement $i$, sur le taux de croissance de l'établissement $i$. Finalement, la somme des deux effets permet ainsi d'obtenir l'effet total.

Une approche différente, et plus simple permet de déterminer les effets directs, indirects et totaux à partir d'une décomposition des paramètres du modèle estimé (Abreu et al., 2005; Dubé \& Legros, 2014; Steimetz, 2010). Cette décomposition consiste à simplifier l'équation 8 afin de se départir de la matrice de pondération spatiale $W$, standardisée en ligne, et de conserver uniquement les paramètres du modèle. Pour ce faire, Kim et al.(2003) démontrent que le multiplicateur spatial, $\left(l_{n}-\rho \mathrm{W}\right)^{-1}$, peut se simplifier pour devenir $(1-\rho)^{-1}$.

La seconde composante de l'équation 8 peut-être décomposée sous forme matricielle:

$$
\left(I_{n} \beta_{k 1}+W \beta_{k 2}\right)=\left[\begin{array}{ccc}
\beta_{k 1} & \cdots & 0 \\
\vdots & \ddots & \vdots \\
0 & \cdots & \beta_{k 1}
\end{array}\right]+\left[\begin{array}{ccc}
w_{11} & \cdots & w_{1 n} \\
\vdots & \ddots & \vdots \\
w_{n 1} & \cdots & w_{n n}
\end{array}\right] \cdot \beta_{k 2}=\left[\begin{array}{ccc}
\beta_{k 1} & \cdots & w_{1 n} \beta_{k 2} \\
\vdots & \ddots & \vdots \\
w_{n 1} \beta_{k 2} & \cdots & \beta_{k 1}
\end{array}\right]
$$

où $w_{i i}=0$, et $\sum_{n=1}^{n} w_{i n}=1$ puisque la matrice $W$ est standardisée en ligne. Ainsi, chaque ligne de l'équation 9, qui correspond à un établissement $i$ se simplifie et s'écrit de la manière suivante:

$$
\beta_{k 1}+\beta_{k 2} \sum_{n=1}^{n} w_{i n}=\beta_{k 1}+\beta_{k 2}
$$

En somme, l'effet total de la variation d'une variable $X_{k}$ se simplifie pour obtenir l'effet marginal total (équation 11).

$$
\partial \Delta e / \partial X_{k}=(1-\rho)^{-1}\left(\beta_{k 1}+\beta_{k 2}\right)
$$

En se basant sur la décomposition de Abreu et al. (2005) et en utilisant le coefficient $\beta_{k 1}$ pour exprimer l'effet direct, alors l'effet margi- nal indirect peut être obtenu comme la différence entre l'effet marginal total et l'effet marginal direct (équation 12).

$$
\left[(1-\rho)^{-1} \cdot\left(\beta_{k 1}+\beta_{k 2}\right)\right]-\beta_{k 1}=(1-\rho)^{-1} \cdot\left(\rho \beta_{k 1}+\beta_{k 2}\right)
$$

À partir de cette approche basée sur la décomposition des paramètres du modèle, il est possible de déterminer la significativité des effets marginaux directs, indirects et totaux grâce à un test de Wald sous les hypothèses suivantes:

Effet direct:

$$
\left.\begin{array}{l}
H_{0}: \beta_{k 1}=0 \\
H_{1}: \beta_{k 1} \neq 0
\end{array}\right\}
$$

Effet indirect:

$$
\left.\begin{array}{l}
H_{0}:(1-\rho)^{-1} \cdot\left(\rho \beta_{k 1}+\beta_{k 2}\right)=0 \\
H_{1}:(1-\rho)^{-1} \cdot\left(\rho \beta_{k 1}+\beta_{k 2}\right) \neq 0
\end{array}\right\}
$$

Effet total:

$$
\left.\begin{array}{l}
H_{0}:(1-\rho)^{-1} \cdot\left(\beta_{k 1}+\beta_{k 2}\right)=0 \\
H_{1}:(1-\rho)^{-1} \cdot\left(\beta_{k 1}+\beta_{k 2}\right) \neq 0
\end{array}\right\}
$$

Ces tests, non linéaires, peuvent être implantés à partir de plusieurs logiciels suite aux estimations des modèles.

\section{DONNÉES}

Les données utilisées proviennent du répertoire LIC (Liste d'Industries et de Commerce) du Ministère de l'Emploi et de la Solidarité (MESS) du Québec. Elles couvrent l'ensemble de la région du BasSaint-Laurent (BSL), une région non métropolitaine dans l'est de la province de Québec. Le BSL n'a aucune ville de plus de 50000 habitants, la limite fixée par Statistique Canada (2015) pour définir une Région Métropolitaine de Recensement (RMR). En 2016, la population de la région s'élevait à 199983 habitants sur une superficie de $22185 \mathrm{~km}^{2}$ avec une densité de 9hab./ $\mathrm{km}^{2}$ (ISQ, 2019). La plus grande ville, Rimouski, compte 48664 habitants avec une densité de 143,3 hab. $/ \mathrm{km}^{2}$. Les autres villes importantes de la région, en termes de population, sont de tailles modestes: Rivière-du-Loup (19 507 hab., 231,9 hab./ $\mathrm{km}^{2}$ ); Matane (14 311 hab., 73 hab. $/ \mathrm{km}^{2}$ ), Amqui (6 178 hab., 51 hab./km2) et La Pocatière (4 120 hab., 190,4 hab./km²).

En raison de son éloignement des grandes régions métropolitaines et de sa faible population, on peut donc qualifier le BSL de région non métropolitaine. La région est située à plus d'une heure de route de la ville de Québec, la région métropolitaine la plus proche, et à plus de cinq heures de route de la ville de Montréal, la plus grande métropole québécoise. Néanmoins, découlant de choix politiques, la région compte une université, située à Rimouski.

Pour fin d'analyse, les microdonnées spatiales longitudinales contiennent 3535 nouveaux établissements apparus entre 2008 et 2014 , dont plus de $80 \%$, soit 2886 établissements, ont survécu jusqu'en 2015. Pour chacun des points (établissements), les informations suivantes sont disponibles: le nombre d'employés en période de pointe, le principal secteur d'activité (code SCIAN - Système de Classification Industrielle d'Amérique du Nord) et la localisation exacte (adresse).

Pour chaque établissement, le taux de croissance de l'emploi durant la période d'observation est considéré comme variable dépendante (équation 1) dans le modèle de croissance.

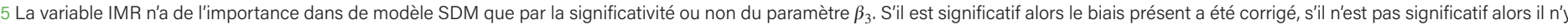
a pas de biais de sélection. De ce fait, son paramètre n'est pas inclus dans la présentation des équations des effets directs/indirects pour simplifier l'écriture. 
Figure 1. Nombre moyen d'employés par secteur d'activité économique selon l'âge

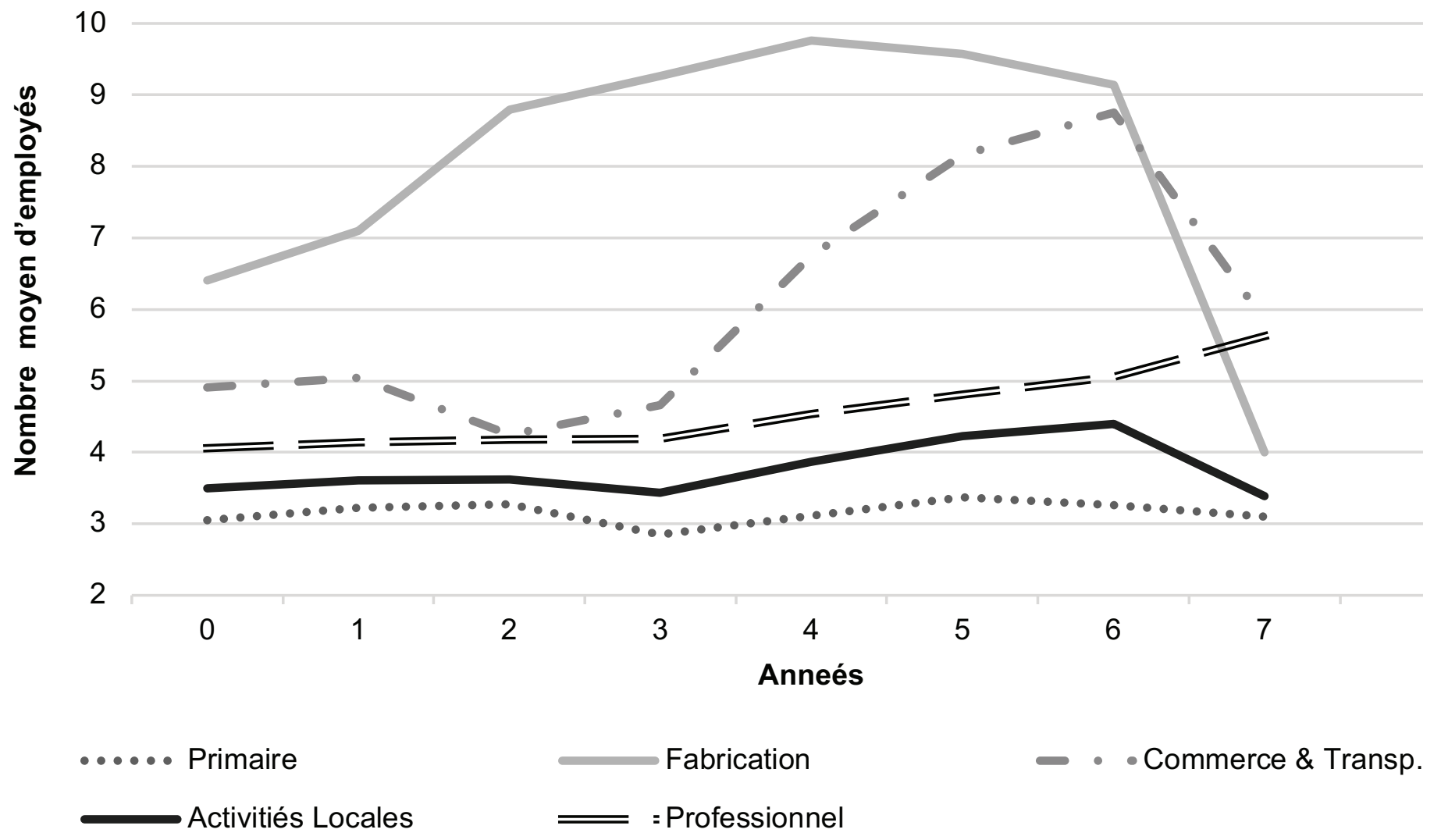

Figure 2. Nombre d'entreprises actives par secteur d'activité économique selon l'âge

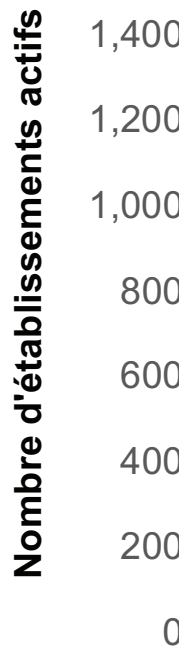

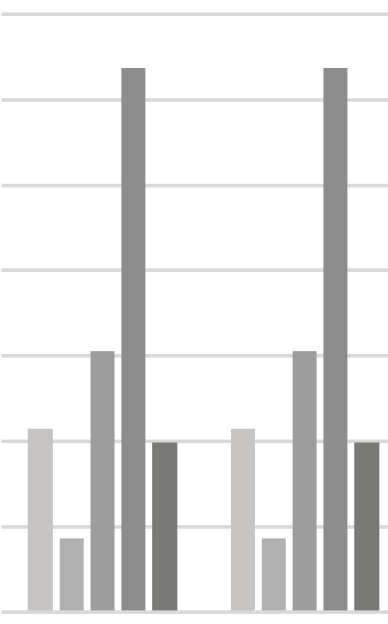

0

1

2

\section{Années}

- Primaire $\quad$ Fabrication $\quad$ Commerce \& Transp. Activitiés Locales $\square$ Professionnel 


\begin{tabular}{|c|c|c|c|}
\hline Secteurs & Sous-Secteurs & Description des Sous-Secteurs & Code SCIAN \\
\hline \multirow{2}{*}{ Primaire } & - Agriculture & Cultures agricoles; Élevage et aquaculture; Activités de soutien aux culturesagricoles/à l'élevage & $\begin{array}{l}111 ; 112 ; \\
1151 ; 1152\end{array}$ \\
\hline & $\begin{array}{l}\text { - Exploitation des ressources } \\
\text { naturelles }\end{array}$ & $\begin{array}{l}\text { Foresterie et exploitation forestière; Pêche, chasse et piégeage; Activités de soutien à la foresterie; } \\
\text { Extraction minière, exploitation en carrière, et extraction de pétrole et de gaz; Transport par pipeline }\end{array}$ & $\begin{array}{l}113 ; 114 ; \\
1153 ; 21 ; 486\end{array}$ \\
\hline Fabrication & - Fabrication & Fabrication & $31 ; 32 ; 33$ \\
\hline \multirow{3}{*}{$\begin{array}{l}\text { Commerce \& } \\
\text { Transport }\end{array}$} & - Commerce de gros & Commerce de gros & 41 \\
\hline & - Commerce de détail & Commerce de détaila & $44 ; 45$ \\
\hline & - Transport & Transport et entreposage ${ }^{b}$ & $48 ; 49$ \\
\hline \multirow{5}{*}{$\begin{array}{l}\text { Activitiés } \\
\text { Locales }\end{array}$} & - Construction & Constructionc & 23 \\
\hline & - Location & $\begin{array}{l}\text { Bailleurs de biens immobiliers; Services de location et de location à bail; } \\
\text { Bailleurs de biens incorporels non financiers }\end{array}$ & $\begin{array}{l}5311 ; 532 \\
533\end{array}$ \\
\hline & - Services de proximité & Magasins d'alimentation; Magasins de produits de santé et de soins personnels; Stations-service & $445 ; 446 ; 447$ \\
\hline & - Hébergement et Restauration & Services d'hébergement et de restauration & 72 \\
\hline & - Autres Services & $\begin{array}{l}\text { Services de soutien d'installations; Services de préparation de voyages et de réservation; Services d'enquêtes } \\
\text { et de sécurité; Services relatifs aux bâtiments et aux logement; Autres services de soutien; Services de gestion } \\
\text { des déchets et d'assainissement; Assistance sociale; Arts, spectacles et loisirs; Autres services }\end{array}$ & $\begin{array}{l}5612 ; 5615 \\
5616 ; 5617 \\
5619 ; 562 ; \\
624 ; 71 ; 81\end{array}$ \\
\hline \multirow{5}{*}{ Professionnel } & - Travaux de génie civil & Travaux de génie civil & 237 \\
\hline & $\begin{array}{l}\text { " Finance Assurance } \\
\text { et Immobilier }\end{array}$ & Finance et assurances; Bureaux d'agents et de courtiers immobiliers; Activités liées à l'immobilier & $\begin{array}{l}52 ; 5312 \\
5313\end{array}$ \\
\hline & $\begin{array}{l}\text { - Services Intensifs } \\
\text { en Connaissances }\end{array}$ & $\begin{array}{l}\text { Éditeurs de logiciels; Traitement de données, hébergement de données et services connexes; } \\
\text { Services professionnels, scientifiques et techniques; Gestion de sociétés et d'entreprises; } \\
\text { Services administratifs de bureau; Services d'emploi; Services de soutien aux entreprises }\end{array}$ & $\begin{array}{l}5112 ; 518 \\
54 ; 55 ; 5611 \\
5613 ; 5614\end{array}$ \\
\hline & - Soins de santé & Cabinets de médecins, dentistes, autres praticiens de la santé; Centres de soins ambulatoires & 621 \\
\hline & $\begin{array}{l}\text { " Industrie de l'information } \\
\text { et industrie culturelle }\end{array}$ & Industrie de l'information et industrie culturelle $e^{e}$ & 51 \\
\hline \multirow[t]{2}{*}{ Public } & - Services Publics & $\begin{array}{l}\text { Bibliothèques; Services d'enseignement; Hôpitaux; Établissements de soins infirmiers et de soins } \\
\text { pour bénéficiaires internes; Organismes religieux, fondations, groupes de citoyens et organisations } \\
\text { professionnelles et similaires; Administrations publiques }\end{array}$ & $\begin{array}{l}519121 ; 61 ; \\
622 ; 623 \\
813 ; 91\end{array}$ \\
\hline & $\begin{array}{l}\text { a Sauf SCIAN } 445,446,447 \\
\text { b Sauf SCIAN } 486 \\
\text { c Sauf SCIAN } 23 \\
\text { d Sauf SCIAN } 8137 \\
\text { e Sauf SCIAN } 5112,518,519121\end{array}$ & & \\
\hline
\end{tabular}

La figure 1 montre l'évolution moyenne du nombre d'employés, selon le secteur, pour les établissements créés entre 2008 et 2014 et qui ont survécus jusqu'en 2015. Dans certains cas, le suivi des établissements peut aller jusqu'a sept (7) ans.

La classification sectorielle utilisée est celle de Cissé et al. (2020), qui utilisent les mêmes données. Au total, six (6) secteurs sont définis à partir du code SCIAN des établissements (Tableau 1): i) le secteur primaire: les activités agricoles, d'élevages et d'exploitation des ressources naturelles; ii) le secteur de la fabrication: la transformation de matière première en produits finis ou semi-finis; iii) le secteur du commerce et du transport; iv) le secteur des activités locales: les services de proximité, la restauration et l'hébergement et les autres services; v) le secteur professionnel: l'ensemble des activités à haute intensité de connaissance et le secteur quaternaire; vi) le secteur public pour toutes les activités à but non lucratif. Par conséquent, la croissance dans ce dernier secteur est surtout exogène et peut dépendre de décisions plutôt politiques.

La région est principalement composée de $\mathrm{PME}$, majoritairement des microentreprises (5 employés et moins). Entre 2008 et 2015, les nouveaux établissements du BSL observent en moyenne une croissance positive du nombre d'employés. Une croissance du nombre moyen d'employés est généralement observée jusqu'à la sixième année. Au-delà de cet âge, tous les secteurs observent une certaine décroissance, sauf pour le secteur professionnel. Les plus faibles va- riations sont observées dans le secteur primaire (3 à 4 employés en moyenne), alors que les plus fortes concernent le secteur de la fabrication et celui du commerce \& du transport.

La figure 2 montre que le nombre de nouveaux établissements qui survivent diminue au fil du temps. À partir de la quatrième année, le taux de survie baisse significativement. Cette observation s'aligne avec plusieurs travaux qui montrent qu'environ la moitié (50\%) des nouvelles entreprises ferment leurs portes dans les cinq premières années de leur existence (Santarelli \& Vivarelli, 2007).

Les variables indépendantes sont divisées en deux groupes (Tableau 2). D'une part, les variables de caractéristiques individuelles: le logarithme de la taille initiale $\ln \left(e_{0}\right)$ c'est-à-dire le logarithme du nombre d'employés à la création, l'âge (Âge) du nouvel établissement en 2015 et enfin, le secteur d'activité économique à la création. L'âge moyen des établissements est d'un plus de trois ans. Les établissements du secteur des activités locales représentent $44,2 \%$ des établissements, alors que les établissements du secteur de la fabrication représentent seulement $6 \%$ des nouveaux établissements de la région.

Pour chaque établissement, les caractéristiques locales sont définies dans un rayon de 1250 mètres. Le choix du rayon est basé sur l'étude de Cissé et al. (2020). Les résultats du modèle de localisation des nouveaux établissements, pour des rayons variant entre 0 


\begin{tabular}{|c|c|c|c|c|}
\hline Variables & Moyenne & Écart-type & $\operatorname{Min}$ & $\operatorname{Max}$ \\
\hline$\Delta \mathrm{e}$ (taux de croissance) & 0.02 & 0.08 & -0.74 & 0.74 \\
\hline \multicolumn{5}{|c|}{ Caractéristiques Individuelles } \\
\hline $\ln \left(e_{0}\right)$ & 0.82 & 0.90 & 0 & 5.52 \\
\hline Âge & 3.33 & 1.86 & 1 & 7 \\
\hline \multicolumn{5}{|l|}{ Caractéristiques Locales } \\
\hline $\ln \left(e_{a}\right)$ & 2.01 & 0.98 & 0 & 5.41 \\
\hline QL Primaire & 1.68 & 3.72 & 0 & 16.15 \\
\hline QL Fabrication & 0.75 & 1.30 & 0 & 8.88 \\
\hline QL Commerce \& Transport & 0.95 & 0.99 & 0 & 7.15 \\
\hline QL Activités Locales & 1.03 & 0.74 & 0 & 4.14 \\
\hline QL Professionnel & 0.91 & 0.95 & 0 & 11.42 \\
\hline QL Publics & 0.80 & 0.70 & 0 & 3.40 \\
\hline $\mathrm{H}_{\mathrm{i}}$ & 0.67 & 0.29 & 0 & 1.00 \\
\hline $\mathrm{RV}_{\mathrm{i}}$ & 1.31 & 0.77 & 0 & 2.26 \\
\hline $\mathrm{C}_{\mathrm{i}}$ & 1.64 & 1.65 & 0 & 11.83 \\
\hline $\mathrm{PM}_{\mathrm{i}}$ & 2.92 & 2.01 & 0.7 & 10.35 \\
\hline
\end{tabular}

\begin{tabular}{lccr}
\hline Variables & Fréquence & Pourcentag \\
\hline S (survivant) & & & \\
& 0 & 649 & 18.4 \\
& 1 & 2,886 & 81.6
\end{tabular}

Caractéristiques Individuelles

Secteur

$\begin{array}{rcc}\text { Primaire } & 430 & 14.9 \\ \text { Fabrication } & 174 & 6.0 \\ \text { Commerce \& Transport } & 611 & 21.2 \\ \text { Activités locales } & 1,275 & 44.2 \\ \text { Professionnel } & 396 & 13.7\end{array}$

Autres

Crise

\begin{tabular}{ccc}
0 & 2,189 & 75.85 \\
1 & 697 & 24.15 \\
\hline
\end{tabular}

$\mathrm{N}$ (survivant) $=2886$

$\mathrm{N}=3535$

et 2 kilomètres, montrent que les résultats issus d'un rayon de 1250 mètres possèdent le pouvoir explicatif (pseudo- $R^{2}$ ) le plus élevé.

Les informations sur les établissements contenus dans ce rayon sont utilisées afin de construire un ensemble d'indicateurs locaux ${ }^{6}$ : i) un quotient de localisation $\left(Q L_{i s}\right)$ de chaque secteur d'activité afin de capturer les effets de co-localisation (spécialisation); ii) l'inverse de l'indice de herfindahl $\left(H_{i}\right)$ pour mesurer la diversité sectorielle locale; iii) $i$ indice d'entropie croisée mesure la variété reliée $\left(R V_{i}\right)$ et capte l'effet de la diversité des activités économiques au sein d'un même secteur; iv) l'indice de concurrence $\left(C_{i}\right)$ de Glaeser et al. (1992); et v) l'indice de potentiel de marché $\left(P M_{i}\right)$, qui exprime l'intensité du contact avec le marché plus précisément la demande intermédiaire extérieure. Puisque l'étude porte sur la croissance de l'emploi des établissements, les quotients de localisation et l'inverse de l'indice de herfindahl sont exprimés en termes de concentration du nombre d'emplois (et non du nombre d'établissements).
La taille des anciens établissements $\left(\ln \left(e_{a}\right)\right)$ dans l'environnement immédiat des nouveaux établissements est aussi prise en compte pour caractériser le milieu. Plus précisément, il s'agit du logarithme du nombre moyen d'employés dans les établissements créés avant 2008 et localisés dans un rayon de 1250 mètres. En moyenne, les anciens établissements sont plus grands que les nouveaux (Tableau 2).

Pour chaque nouvel établissement, ces indices définissent les caractéristiques locales une année avant la date de création. L'exercice a aussi été réalisé avec un décalage temporel de deux années. Le décalage temporel permet, d'une part, d'éviter les problèmes d'endogénéités et, d'autre part, de tester la robustesse des résultats d'autant plus que la période d'étude comporte une période de récession.?

La proximité aux principales villes de la région est contrôlée à travers la distance (en logarithme) entre chaque établissement et le centreville des principales villes. Ces distances servent aussi de proxy pour contrôler l'effet d'urbanisation.

Enfin, l'effet de la récession économique entre 2008 et 2010 est pris en compte dans l'étude à travers une variable binaire (Crise). Elle prend la valeur 1 lorsque l'établissement est actif durant au moins une année sur les trois années de la récession. Pour tenir compte de la période de récession, des variables d'interaction ont été créées entre la variable (Crise) et chaque variable indépendante. Les résultats des différentes estimations sont présentés en deux périodes: une période de récession issue des variables d'interactions et une période post-récession ou une période normale.

\section{RÉSULTATS}

\section{Modèle de sélection probit}

Le Tableau 3 présente les résultats du modèle de sélection qui sont globalement significatifs, comme le montre le test de $x^{2}$. Pour ce qui est des caractéristiques individuelles, seulement l'âge augmente la probabilité de survie des nouveaux établissements durant la période de récession, alors que leurs probabilités de survie augmentent avec la taille et l'âge pendant la période post-récession.

Les nouveaux établissements du secteur primaire et ceux du secteur du commerce \& du transport ont une plus faible probabilité de survie que ceux du secteur des activités locales (la catégorie de référence). Par contre, en période de récession, les nouveaux établissements du secteur du commerce \& du transport et ceux du secteur de la fabrication sont plus résilients que les nouveaux établissements actifs dans les activités locales.

Au niveau des caractéristiques locales, en période de récession, c'est la diversité entre les secteurs, la concentration d'activité dans le secteur du commerce \& du transport et la concentration entre les établissements (spécialisation) dans les activités locales, qui favorisent la survie des nouveaux établissements. La résilience des nouveaux établissements dans le secteur de la fabrication face à la récession est mise à l'épreuve lorsque ces derniers sont localisés dans un milieu fortement concentré en établissements du même secteur.

En période post-récession, la taille des anciens établissements et la demande intermédiaire extérieure augmentent la probabilité de survie des nouveaux établissements dans le BSL tandis que la diversité intersectorielle (variété reliée) diminue cette probabilité.

Quant aux effets d'urbanisation, l'impact reste le même, peu importe la période; plus le nouvel établissement est proche des grandes villes de la région, plus sa probabilité de survie augmente. 
Tableau 3. Résultat de l'estimation de modèles de sélection PROBIT

Récession Post-recession

$\begin{array}{llllll}\text { Variables Coef. Std.Err. } & z & P>z & \text { Coef. Std.Err. } & z & P>z\end{array}$ Caracteristiques Individuelles

$\begin{array}{lccccccccc}\text { In }\left(\mathrm{e}_{0}\right) & -0.05 & 0.13 & -0.4 & & 0.18 & 0.06 & 3.2 & * * * \\ \text { Âge } & 1.05 & 0.13 & 7.8 & * * * & 0.72 & 0.05 & 15.9 & * * * \\ \text { Secteur } & & & & & & & & \\ \text { Primaire } & 0.22 & 0.51 & 0.4 & & -0.51 & 0.24 & -2.1 & * * \\ \text { Fabrication } & 1.12 & 0.49 & 2.3 & * * & -0.19 & 0.27 & -0.7 & \\ \text { Commerce \& Transp. } & 0.88 & 0.50 & 1.8 & * & -0.48 & 0.22 & -2.2 & * * \\ \text { Professionnel } & 0.56 & 0.52 & 1.1 & & -0.06 & 0.25 & -0.2 & \end{array}$

Caracteristiques Locales ( $t-1)$

\begin{tabular}{|c|c|c|c|c|c|c|c|c|}
\hline $\ln \left(e_{a}\right)$ & -0.30 & 0.23 & -1.3 & & 0.31 & 0.10 & 3.1 & $* * *$ \\
\hline QL Primaire & 0.06 & 0.06 & 1.1 & & -0.04 & 0.03 & -1.3 & \\
\hline QL Fabrication & 0.06 & 0.11 & 0.6 & & -0.03 & 0.05 & -0.7 & \\
\hline $\begin{array}{l}\text { QL Commerce \& } \\
\text { Transp. }\end{array}$ & 0.31 & 0.15 & 2.0 & ** & -0.07 & 0.07 & -1.0 & \\
\hline QL Activités Locales & -0.17 & 0.29 & -0.6 & & 0.11 & 0.14 & 0.8 & \\
\hline QL Professionnel & 0.01 & 0.13 & 0.1 & & -0.02 & 0.06 & -0.3 & \\
\hline QL Publics & 0.12 & 0.29 & 0.4 & & -0.17 & 0.12 & -1.4 & \\
\hline $\mathrm{H}_{\mathrm{i}}$ & 0.99 & 0.51 & 1.9 & * & -0.22 & 0.28 & -0.8 & \\
\hline $\mathrm{RV}_{\mathrm{i}}$ & -0.03 & 0.29 & -0.1 & & -0.31 & 0.14 & -2.3 & ** \\
\hline $\mathrm{C}_{\mathrm{i}}$ & -0.12 & 0.12 & -1.0 & & 0.06 & 0.05 & 1.2 & \\
\hline $\mathrm{PM}_{\mathrm{i}}$ & -0.07 & 0.11 & -0.6 & & 0.09 & 0.04 & 2.1 & ** \\
\hline Crise & & & & & -3.58 & 2.29 & -1.6 & \\
\hline QL Primaire $x$ Primaire & -0.02 & 0.06 & -0.3 & & 0.02 & 0.03 & 0.7 & \\
\hline $\begin{array}{l}\text { QL Fabrication x } \\
\text { Fabrication }\end{array}$ & -0.57 & 0.26 & -2.2 & $* *$ & 0.04 & 0.16 & 0.3 & \\
\hline $\begin{array}{l}\text { QL Commerce \& Trans. } \\
\text { x Commerce \& Transp. }\end{array}$ & -0.34 & 0.23 & -1.5 & & 0.06 & 0.10 & 0.6 & \\
\hline $\begin{array}{l}\text { QL Activités Locales x } \\
\text { Activités Locales }\end{array}$ & 0.48 & 0.25 & 1.9 & * & -0.11 & 0.15 & -0.8 & \\
\hline $\begin{array}{l}\text { QL Professionnel x } \\
\text { Professionnel }\end{array}$ & -0.38 & 0.23 & -1.6 & & -0.09 & 0.14 & -0.7 & \\
\hline $\begin{array}{l}\text { Distance à Rimouski } \\
\text { Àen km (log) }\end{array}$ & 0.02 & 0.13 & 0.2 & & -0.05 & 0.06 & -0.9 & \\
\hline $\begin{array}{l}\text { Distance à Rivière-du- } \\
\text { Loup en km (log) }\end{array}$ & -0.27 & 0.13 & -2.1 & ** & -0.10 & 0.05 & -1.8 & * \\
\hline $\begin{array}{l}\text { Distance à Matane } \\
\text { en km (log) }\end{array}$ & -0.26 & 0.14 & -1.8 & * & -0.09 & 0.04 & -2.1 & ** \\
\hline $\begin{array}{l}\text { Distance à Amqui } \\
\text { en km (log) }\end{array}$ & -0.12 & 0.14 & -0.9 & & -0.06 & 0.05 & -1.0 & \\
\hline $\begin{array}{l}\text { Distance à La Pocatière } \\
\text { en } \mathrm{km}(\mathrm{log})\end{array}$ & -0.46 & 0.11 & -4.1 & $* * *$ & -0.09 & 0.05 & -1.9 & * \\
\hline Pseudo $\mathrm{R}^{2}$ & & 0.63 & & & & & & \\
\hline Wald X2 (55) & & 828.25 & & $* * *$ & & & & \\
\hline $\mathrm{N}$ & & 3535 & & & & & & \\
\hline
\end{tabular}

Légende: ${ }^{* * *} p<1 \% ;{ }^{* *} p<5 \% ;{ }^{*} p<10 \%$

\section{Modèle de croissance SDM}

Pour s'assurer de la validité des résultats, l'une des conditions des modèles spatiaux est que la matrice de pondération spatiale mette en relation chacune des observations avec au moins une autre observation. Pour répondre à cette exigence, la matrice de pondération spatiale (W) est construite sur la base des «plus proches observations », Le terme «observation » est préféré au terme «voisin» dans

\section{Tableau 4. Matrices de pondérations spatiales}

\begin{tabular}{lcccc}
\multicolumn{1}{c}{$\begin{array}{c}\text { Matrice de } \\
\text { pondération spatiale }\end{array}$} & $\begin{array}{c}\text { Distance moyenne } \\
\text { (en mètre) }\end{array}$ & Min. & Moyenne & Max. \\
\hline $\begin{array}{l}5 \text { plus proches } \\
\text { observations } \\
\begin{array}{l}10 \text { plus proches } \\
\text { observations }\end{array}\end{array}$ & 899.9 & 5 & 6 & 30 \\
$\begin{array}{l}15 \text { plus proches } \\
\text { observations }\end{array}$ & 1354.8 & 10 & 12 & 39 \\
$\begin{array}{l}20 \text { plus proches } \\
\text { observations }\end{array}$ & 1764.2 & 15 & 18 & 46 \\
\hline
\end{tabular}

l'optique où plusieurs établissements peuvent partager une même localisation (un centre commercial, par exemple). Il est donc possible que les plus faibles distances permettent d'isoler un nombre plus grand de relations spatiales. En effet, la matrice des 5 plus faibles distances (ou plus proches observations) peut mettre en relation jusqu'à 30 établissements et la distance moyenne entre un nouvel établissement et ses plus proches observations, d'autres nouveaux établissements, est de 899,9 mètres (Tableau 4). Afin de tester la robustesse des résultats, plusieurs matrices de pondérations spatiales, standardisées en ligne, ont été construites en faisant varier (5 à 20) le nombre de plus proches observations.

Le tableau 5 présente les résultats des coefficients des modèles avec les 5 et 20 plus proches observations tandis que les effets directs, indirects et totaux avec ces mêmes matrices de pondérations spatiales sont présentés dans le Tableau 6.

Les résultats montrent l'importance de contrôler pour le possible biais de sélection (ou d'attrition) puisque le coefficient lié à l'inverse du ratio de Mills (IRM) est significatif dans chaque estimation (Tableau 5).

Les résultats suggèrent également que les effets de débordements spatiaux ne sont pas globaux (coefficient lié à la variable dépendante décalée spatialement), mais plutôt locaux (coefficients liés aux variables indépendantes décalées spatialement). Le coefficient de dépendance spatiale de la variable dépendante n'est pas significatif, ce qui indique que les effets de débordements spatiaux globaux n'impactent que marginalement les effets indirects et totaux. Les effets de débordements locaux spatiaux sont néanmoins minimisés par le fait que seul le coefficient lié au secteur du commerce \& du transport est significatif en période de récession.

Bien que le tableau des coefficients revête un certain intérêt, la particularité du modèle SDM fait en sorte que l'interprétation est complète lorsque I'on utilise la décomposition des effets marginaux (Tableau 6). L'âge et la taille des nouveaux établissements se présentent comme les caractéristiques individuelles qui influencent, de façon différente, la croissance de l'emploi des nouveaux établissements. En effet, dans la région du BSL pour un nouvel établissement, alors que l'âge est un facteur qui contribue à sa croissance en période normale, son taux de croissance de l'emploi diminue avec l'âge en période de récession.

Les résultats suggèrent, avec la matrice des 5 plus proches observations, qu'une augmentation de $1 \%$ de l'âge d'un nouvel établissement augmente de $0,042 \%$ son taux de croissance de l'emploi (effet direct) en période normale. Pour un nouvel établissement d'âge moyen (3,3 ans), une année (ou 30,3\%) de plus entraîne, toute chose étant égale par ailleurs, une augmentation de 1,27\% de son taux de croissance de l'emploi. L'effet total est le même puisque l'effet indirect est non significatif. En période de récession, les résultats sont plus en lien avec la littérature qui veut que la croissance de l'entreprise 
Tableau 5. Coefficients de l'estimation des modèles SDM

5 plus proches observations

20 plus proches observations

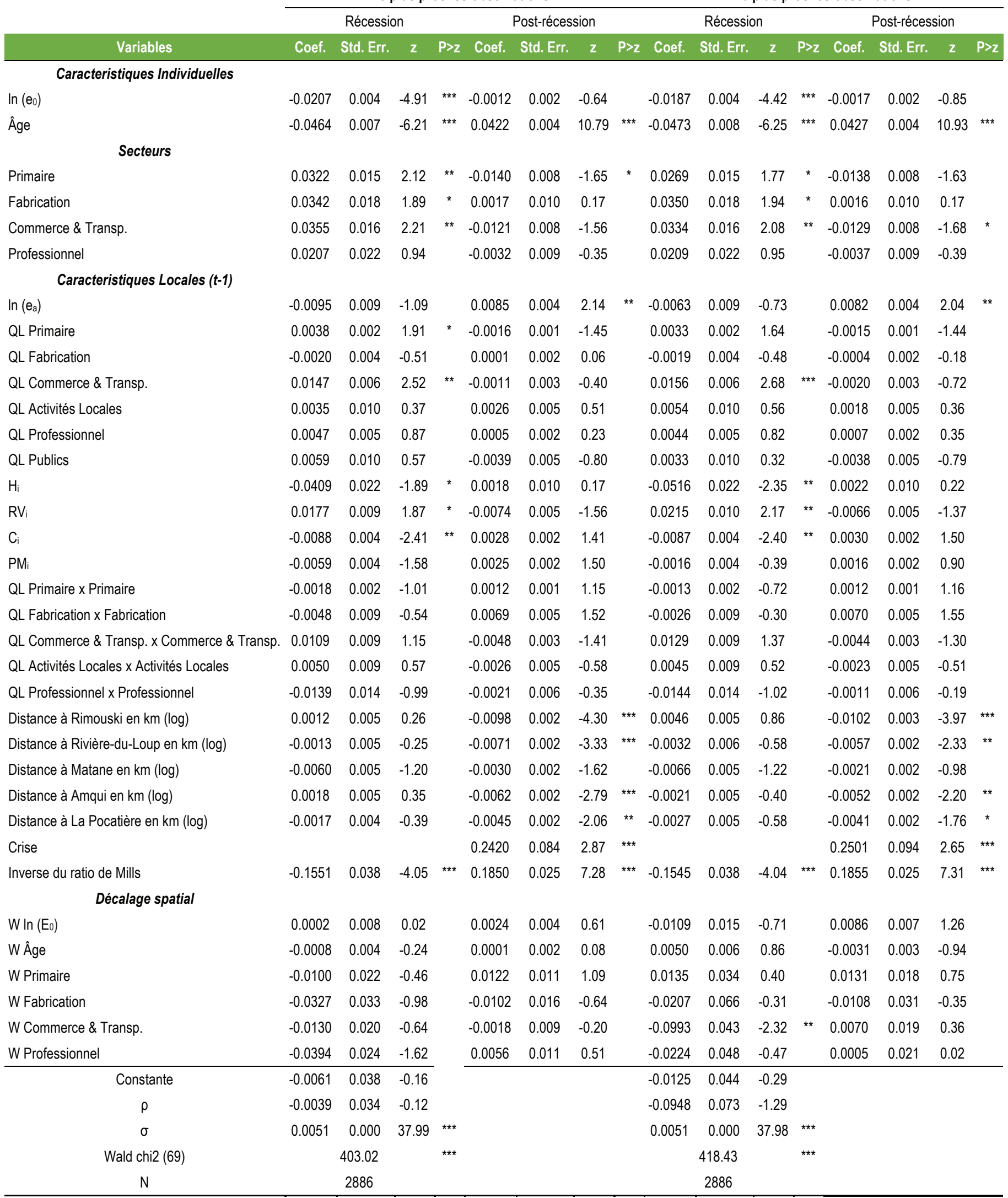

Légende: ${ }^{* * *} p<1 \% ;{ }^{* *} p<5 \% ;{ }^{*} p<10 \%$ 
Tableau 6. Impact des changements des variables explicatives: les effets

5 plus proches observations

20 plus proches observations

\begin{tabular}{|c|c|c|c|c|c|c|c|c|c|c|c|c|c|c|c|c|c|c|c|c|c|c|c|}
\hline \multirow[b]{2}{*}{ Variables } & \multicolumn{5}{|c|}{ Récession } & \multicolumn{6}{|c|}{ Post récession } & \multicolumn{6}{|c|}{ Récession } & \multicolumn{6}{|c|}{ Post récession } \\
\hline & $\begin{array}{l}\text { Effects } \\
\text { directs }\end{array}$ & Sign. & Effects Sign. & Effects & Sign. & $\begin{array}{l}\text { Effects } \\
\text { directs }\end{array}$ & Sign. & $\begin{array}{l}\text { Effects } \\
\text { indirects }\end{array}$ & Sign. & Effects & Sign. & $\begin{array}{l}\text { Effects } \\
\text { directs }\end{array}$ & Sign. & $\begin{array}{l}\text { Effects } \\
\text { indirects }\end{array}$ & Sign. & Effects & Sign. & $\begin{array}{l}\text { Effects } \\
\text { directs }\end{array}$ & Sign. & $\begin{array}{l}\text { Effects } \\
\text { indirects }\end{array}$ & Sign. & Effects & Sign. \\
\hline \multicolumn{24}{|c|}{ Caractéristiques Individuelles } \\
\hline $\ln \left(\mathrm{e}_{0}\right)$ & -0.0207 & $* * *$ & 0.0003 & -0.0205 & $*$ & -0.0012 & & 0.0024 & & 0.0012 & & -0.0187 & $* * *$ & -0.0084 & & -0.0271 & & -0.0017 & & 0.0080 & & 0.0063 & \\
\hline Âge & -0.0464 & $* * *$ & -0.0006 & -0.0470 & $* \star \star$ & 0.0422 & $* \star \star$ & 0.0000 & & 0.0422 & $* * \star$ & -0.0473 & $* \star \star$ & 0.0087 & & -0.0387 & $* * *$ & 0.0427 & $* \star \star$ & -0.0065 & * & 0.0361 & $* * *$ \\
\hline \multicolumn{24}{|l|}{ Secteurs } \\
\hline Fabrication & 0.0342 & & -0.0327 & 0.0015 & & 0.0017 & & -0.0101 & & -0.0084 & & 0.0350 & & -0.0220 & & 0.0130 & & 0.0016 & & -0.0100 & & -0.0084 & \\
\hline Commerce \& Transp. & 0.0355 & * & -0.0131 & 0.0224 & & -0.0121 & & -0.0018 & & -0.0138 & & 0.0334 & * & -0.0936 & * & -0.0602 & & -0.0129 & & 0.0075 & & -0.0055 & \\
\hline Professionnel & 0.0207 & & -0.0393 & -0.0186 & & -0.0032 & & 0.0056 & & 0.0024 & & 0.0209 & & -0.0223 & & -0.0014 & & -0.0037 & & 0.0008 & & -0.0029 & \\
\hline \multicolumn{24}{|l|}{ Caracteristiques Locales (t-1) } \\
\hline $\ln \left(e_{a}\right)$ & -0.0095 & & 0.0000 & -0.0095 & & 0.0085 & $* *$ & 0.0000 & * & 0.0084 & * & -0.0063 & & 0.0005 & & -0.0058 & & 0.0082 & * & -0.0007 & * & 0.0075 & * \\
\hline QL Activités Locales & 0.0035 & & 0.0000 & 0.0035 & & 0.0026 & & 0.0000 & & 0.0026 & & 0.0054 & & -0.0005 & & 0.0049 & & 0.0018 & & -0.0002 & & 0.0016 & \\
\hline QL Professionnel & 0.0047 & & 0.0000 & 0.0047 & & 0.0005 & & 0.0000 & & 0.0005 & & 0.0044 & & -0.0004 & & 0.0040 & & 0.0007 & & -0.0001 & & 0.0007 & \\
\hline QL Publics & 0.0059 & & 0.0000 & 0.0059 & & -0.0039 & & 0.0000 & & -0.0039 & & 0.0033 & & -0.0003 & & 0.0030 & & -0.0038 & & 0.0003 & & -0.0035 & \\
\hline $\mathrm{H}_{\mathrm{i}}$ & -0.0409 & & 0.0002 & -0.0407 & & 0.0018 & & 0.0000 & & 0.0018 & & -0.0516 & * & 0.0045 & * & -0.0471 & * & 0.0022 & & -0.0002 & & 0.0021 & \\
\hline $\mathrm{RV}_{\mathrm{i}}$ & 0.0177 & & -0.0001 & 0.0176 & & -0.0074 & & 0.0000 & & -0.0073 & & 0.0215 & * & -0.0019 & * & 0.0196 & * & -0.0066 & & 0.0006 & & -0.0060 & \\
\hline $\mathrm{C}_{\mathrm{i}}$ & -0.0088 & * & $0.0000 *$ & -0.0087 & * & 0.0028 & & 0.0000 & & 0.0028 & & -0.0087 & * & 0.0008 & * & -0.0080 & * & 0.0030 & & -0.0003 & & 0.0027 & \\
\hline $\mathrm{PM}_{\mathrm{i}}$ & -0.0059 & & 0.0000 & -0.0059 & & 0.0025 & & 0.0000 & & 0.0025 & & -0.0016 & & 0.0001 & & -0.0014 & & 0.0016 & & -0.0001 & & 0.0014 & \\
\hline QL Primaire $x$ Primaire & -0.0018 & & 0.0000 & -0.0018 & & 0.0012 & & 0.0000 & & 0.0012 & & -0.0013 & & 0.0001 & & -0.0012 & & 0.0012 & & -0.0001 & & 0.0011 & \\
\hline QL Fabrication x Fabrication & -0.0048 & & 0.0000 & -0.0048 & & 0.0069 & & 0.0000 & & 0.0069 & & -0.0026 & & 0.0002 & & -0.0024 & & 0.0070 & & -0.0006 & & 0.0064 & \\
\hline $\begin{array}{l}\text { Distance à Rivière- } \\
\text { du-Loup en km (log) }\end{array}$ & -0.0013 & & 0.0000 & -0.0013 & & -0.0071 & $* * *$ & 0.0000 & $* * *$ & -0.0071 & $* * *$ & -0.0032 & & 0.0003 & & -0.0029 & & -0.0057 & * & 0.0005 & * & -0.0052 & * \\
\hline $\begin{array}{l}\text { Distance à Matane } \\
\text { en km (log) }\end{array}$ & -0.0060 & & 0.0000 & -0.0060 & & -0.0030 & & 0.0000 & & -0.0030 & & -0.0066 & & 0.0006 & & -0.0060 & & -0.0021 & & 0.0002 & & -0.0019 & \\
\hline $\begin{array}{l}\text { Distance à Amqui } \\
\text { en km (log) }\end{array}$ & 0.0018 & & 0.0000 & 0.0018 & & -0.0062 & $* * *$ & 0.0000 & ** & -0.0061 & ** & -0.0021 & & 0.0002 & & -0.0020 & & -0.0052 & * & 0.0004 & * & -0.0047 & * \\
\hline $\begin{array}{l}\text { Distance à La Pocatière } \\
\text { en km (log) }\end{array}$ & -0.0017 & & 0.0000 & -0.0017 & & -0.0045 & ** & 0.0000 & * & -0.0044 & * & -0.0027 & & 0.0002 & & -0.0025 & & -0.0041 & & 0.0004 & & -0.0038 & \\
\hline Crise & & & & & & 0.2420 & $* \star *$ & -0.0009 & ** & 0.2411 & $* *$ & & & & & & & 0.2501 & ** & -0.0217 & ** & 0.2285 & ** \\
\hline
\end{tabular}

Légende: ${ }^{* * *} p<1 \% ;{ }^{* *} p<5 \% ;{ }^{*} p<10 \%$ 
tende à diminuer avec l'âge (David B. Audretsch \& Dohse, 2007). Une augmentation de $1 \%$ de l'âge (ou 1 an) d'un nouvel établissement diminue de $0,046 \%$ (1,4\%) son taux de croissance de l'emploi (effet direct).

Quant à la taille, elle a un impact négatif sur la croissance de l'emploi des nouveaux établissements durant la récession. Une augmentation de $1 \%$ de la taille d'un nouvel établissement a pour effet, direct et total, de diminuer de $0,02 \%$ son taux de croissance de l'emploi. Ainsi, l'effet de l'âge est en partie contrebalancé par l'effet de la taille, qui suggère que la croissance de l'emploi est limitée (et non exponentielle). Ces résultats restent constants et assez similaires lorsqu'on fait varier le nombre de proches observations à considérer contrairement au secteur d'activité qui est plus ou moins sensible au nombre de plus proches observations.

Lorsqu'on considère les 5 plus proches observations, le taux de croissance de l'emploi des nouveaux établissements agricoles est plus faible $(0,014 \%)$ que celui des nouveaux établissements des activités locales en période normale, et l'inverse est constaté en période de récession. Par contre, lorsqu'on considère les 20 plus proches observations, ce sont les nouveaux établissements dans le secteur du commerce \& du transport qui se démarquent en période de récession avec un taux de croissance de l'emploi plus élevé (0,033\%) que celui des nouveaux établissements du secteur des activités locales. Cependant, pour un nouvel établissement, avoir ses plus proches observations issues du secteur du commerce \& du transport au lieu du secteur des activités locales, diminue de 0,09\% son taux de croissance de l'emploi (effet indirect).

Pour ce qui est des caractéristiques locales, leurs impacts sont plus visibles en période de récession, à l'exception de la taille des anciens établissements du milieu. En effet, en période normale, une augmentation de $1 \%$ de la taille des établissements créés avant 2008 (anciens établissements) et localisés dans le rayon de 1250 mètres autour du nouvel établissement augmente de 0,008\% le taux de croissance de l'emploi du nouvel établissement. Lorsqu'un nouvel établissement en général ou du secteur du commerce \& du transport en particulier est localisé dans un milieu concentré en activité du secteur du commerce \& du transport, cela a un effet positif sur le taux de croissance de l'emploi de ce nouvel établissement en période de récession. Dans un rayon de 1250 mètres (effet direct), une augmentation de $1 \%$ de la concentration d'établissements du secteur du commerce \& du transport augmente de 0,014\% le taux de croissance de l'emploi d'un nouvel établissement, en général, et de $0,010 \%$ si ce dernier est actif dans le secteur du commerce \& du transport. Au-delà de 1250 mètres (effet indirect), une augmentation (1\%) de la concentration d'établissements du secteur du commerce \& du transport diminue de façon marginale le taux de croissance de l'emploi du nouvel établissement. De façon générale, l'effet total est positif et se traduit par une augmentation de 0,014\% du taux de croissance de l'emploi lorsque la concentration d'établissements du secteur du commerce \& du transport augmente de 1\% dans l'ensemble de la région en période de récession.

La diversification et la concurrence diminuent le taux de croissance de l'emploi des nouveaux établissements en période de récession. Lorsque chacune de ces variables augmente de 1\%, la baisse du taux de croissance de l'emploi des nouveaux établissements est estimée à 0,05\% du fait de la diversification et à 0,0088\% du fait de la concurrence. L'effet total de ces variables reste sensiblement pareil à celui de l'effet direct puisque l'effet indirect, bien que significatif, reste très faible. Enfin, pour une augmentation de $1 \%$ de la variété reliée, le taux de croissance de l'emploi des nouveaux établissements durant la récession augmente de 0,02\%. II faut noter que les effets de diversification et la variété reliée sont sensibles à la matrice de pondération utilisée.
L'effet d'urbanisation, à travers la proximité aux principales villes de la région: Rimouski, Rivière-du-Loup et Amqui, est un facteur qui favorise la croissance du taux de l'emploi des nouveaux établissements en période post-récession. En effet, plus le nouvel établissement est proche de l'une de ces villes, plus grande sera la croissance de son taux d'emploi (effet direct). Les effets indirects, bien que significatifs, restent très faibles. Ainsi, l'effet total de la proximité à ces trois villes de la région à un impact positif sur la croissance de l'emploi des nouveaux établissements en période post-récession.

\section{DISCUSSION}

Les résultats du modèle de croissance régionale adapté aux nouveaux établissements sont peu sensibles au choix de la matrice de pondération spatiale et au décalage temporel (annexe 2 et 3), ce qui confirme la robustesse des résultats de façon générale.

La correction pour la présence de biais de sélection a permis de déterminer les facteurs de la survie des nouveaux établissements et de constater que la notion de performance d'entreprise est relativement sensible aux réalités locales. En effet, les caractéristiques individuelles et locales susceptibles, selon la littérature, de favoriser la croissance des entreprises se sont révélées, en majorité, significatives pour la survie des nouveaux établissements dans la région du Bas-Saint-Laurent aussi bien en période normale que durant la récession. Les travaux de Coad \& Broekel (2012) montrent que l'augmentation du taux d'emploi est en général associée à une diminution de la productivité alors que les entreprises qui ont une forte croissance de productivité peuvent en général avoir une croissance de l'emploi plus importante que les entreprises à plus faible productivité. Cela amène à croire que l'objectif des établissements en région éloignée, comme pour le BSL, pourrait être de s'assurer une présence stable dans le temps plutôt que de rechercher une forte croissance (des «gazelles»). De ce fait, cette situation doit être considérée par les décideurs locaux dans l'élaboration de politique de création et soutien au développement des entreprises. La recherche de la pérennité peut alors primer pour ces régions, par opposition à la recherche d'une croissance soutenue de l'emploi.

Les effets indirects des caractéristiques individuelles n'ont pas, pour la plupart, d'impact sur la croissance du taux d'emploi des nouveaux établissements de la région, et ce peu importe la matrice considérée. La seule exception au tableau vient de la variable de l'âge et du secteur du commerce \& du transport. Les effets directs représentent au moins $90 \%$ des effets totaux pour chacune des caractéristiques (individuelle et locale) dont l'impact est significatif. Comme les travaux de Garsaa \& Levratto (2017), ces résultats montrent la prédominance des attributs propres à chaque établissement sur le taux de croissance de l'emploi d'autant plus que chacune des caractéristiques individuelles testées par le modèle explique de façon significative la croissance de l'emploi des nouveaux établissements, du moins dans une région non métropolitaine. L'indépendance aux sources de financement externe des petites entreprises en région éloignée pourrait expliquer la relation inverse entre la taille et la croissance durant la récession (Bricogne et al., 2012).

En observant les résultats des caractéristiques locales, en général, on peut retenir que c'est en période de récession que le milieu fait la différence. En effet, la spécialisation industrielle relative au secteur du commerce \& du transport et la variété reliée se présentent comme des milieux favorables à la croissance de l'emploi des nouveaux établissements en période de récession. Par ailleurs, l'impact négatif des effets indirects (au-delà du 1250 mètres) de ces variables sur le taux de la croissance de l'emploi reflète l'avantage comparatif que peut posséder une localité spécialisée. En d'autres termes, toute l'étendue la région du BSL n'est pas concentrée (spécialisée) 
dans le secteur du commerce \& du transport ou dans un ensemble de sous secteurs, ce qui favorise donc les localités qui le sont. Par ailleurs, même si les effets d'agglomérations attirent les établissements vers certaines localités du fait des avantages qu'ils présentent, une trop forte concentration d'activités économiques peut produire par la même occasion des externalités négatives. D'où l'impact négatif de la spécialisation au-delà 1250 mètres. En somme, ils démontrent que le «bassin d'emploi » est relativement fixe, ainsi la croissance de l'emploi de nouveaux établissements peut être limitée par le nombre de nouveaux établissements qui entrent sur le marché.

Comme le mentionnent Macpherson \& Holt (2007), la croissance des petites entreprises est de nature complexe et idiosyncrasique ce qui peut expliquer en partie le faible impact des variables sélectionnées dans le modèle. De plus, les nouveaux établissements sont, pour la plupart, de très petits établissements, voire des établissements individuels. La croissance de l'emploi, la variable dépendante, varie très peu pour ce type d'entreprises, encore plus en région non métropolitaine.

En comparaison avec l'étude de Cissé et al. (2020), on peut déduire que les caractéristiques locales en termes de concentration sectorielle contribuent moins à la croissance de l'emploi par rapport à la création d'établissements dans la région du BSL. Cependant, des politiques locales de subventions par exemple pourraient servir de stabilisateur en assurant la croissance et la survie des établissements en région non métropolitaine particulièrement en période de récession (Belleau-Arsenault \& Dubé, 2019).

Toutefois, même si les caractéristiques locales ont un impact relativement faible, l'effet de proximité (les plus faibles distances aux établissements ou ceux dans un rayon de 1250 mètres) explique en partie le taux de croissance de l'emploi des nouveaux établissements dans la région du BSL.

\section{CONCLUSION}

À partir de microdonnées spatiales, cet article utilise un modèle de croissance spatiale appliqué aux nouveaux établissements afin de vérifier si des caractéristiques individuelles et/ou locales ont un impact sur la croissance de l'emploi des nouveaux établissements. L'étude de cas porte sur les établissements créés entre 2008 et 2014 dans la région non métropolitaine du Bas-Saint-Laurent (BSL) au Québec. À partir de la localisation exacte de chaque établissement, des indicateurs locaux sont construits, afin de capter des effets de débordements locaux, alors que l'inclusion du décalage spatial de la variable dépendante permet de contrôler pour les effets de débordements spatiaux globaux. Malgré le peu de variabilité dans la croissance de l'emploi des nouveaux établissements, le modèle statistique permet d'identifier des facteurs (individuels et locaux) qui influencent de manière significative la croissance de l'emploi des nouveaux établissements.

Les résultats du modèle spatial de type SDM-Spatial Durbin Model, après avoir corrigé le biais de sélection, peuvent être classés dans un ordre décroissant selon l'intensité des impacts (effet direct, indirect et total). D'abord, la grande récession économique de 2008, dont le coefficient positif révèle l'opportunité saisie par les nouveaux établissements. Puisque la période d'étude commence par l'année 2008, l'année du début de la crise, les nouveaux établissements qui ont osé démarrer leurs activités dans ce contexte ont certainement anticipé de pouvoir tirer parti de ce même contexte. Elle est suivie de loin par l'âge des nouveaux établissements dont l'impact sur la croissance de l'emploi des nouveaux établissements est positif en période post-récession, mais négatif en période de récession. Ensuite, la taille des nouveaux établissements suggère un effet négatif sur le taux de croissance de l'emploi des nouveaux établissements en période de récession. Enfin, l'impact positif de la spécialisation industrielle relative au secteur du commerce \& du transport sur la croissance de l'emploi des nouveaux établissements. Finalement, l'effet d'urbanisation favorise la croissance par la proximité aux principales villes, soit Rimouski, Rivière-du-Loup et Amqui.

D'un point de vue méthodologique, l'originalité de cet article réside dans l'application d'un modèle de croissances à une échelle micro: les nouveaux établissements. La décomposition des paramètres du modèle spatial pour calculer les effets directs, indirects et totaux, mais surtout la proposition des tests de significativité, issus de tests non linéaires, s'avère être également une originalité. Cette approche, à travers la mise en œuvre du test de Wald, permet d'adopter une approche relativement simple et intuitive par rapport à celle proposée par LeSage \& Pace (2009), qui restent avant tout exigeants en temps et en mémoire informatique.

Quelques limites ressortent de cette analyse principalement en ce qui concerne la base de données. Elle a l'avantage de fournir des informations géolocalisées qui permettent de caractériser le milieu. Par contre, elle a l'inconvénient de fournir trop peu d'informations sur les caractéristiques individuelles des nouveaux établissements et celles des entrepreneurs. Des informations additionnelles telles que le chiffre d'affaires, les fusions ou acquisitions, le profil des entrepreneurs ou des gestionnaires sont autant de caractéristiques qui peuvent être intégrées dans l'analyse de la croissance individuelle des entreprises. II en est de même pour les caractéristiques des anciens établissements (tailles, secteurs d'activité) à proximité des nouveaux établissements. Bien que l'approche par le modèle de type SDM-Spatial Durbin Model permet de contrôler pour divers facteurs et l'omission de certains facteurs importants, l'analyse pourrait être bonifiée avec des données sur des caractéristiques locales telles que les niveaux des salaires, le cout immobilier et les caractéristiques des ménages afin de mieux connaître l'environnement socioéconomique des nouveaux établissements.

\section{BIBLIOGRAPHIE}

Abreu, M., de Groot, H. L. F., \& Florax, R. J. G. M. (2005). Space and Growth: A Survey of Empirical Evidence and Methods. SSRN Electronic Journal.

Acosta, S. A., Crespo, H. Á., \& Agudo, C. J. (2018). Effect of market orientation, network capability and entrepreneurial orientation on international performance of small and medium enterprises (SMEs). International Business Review, 27(6), 1128-1140.

Audretsch, David B., \& Dohse, D. (2007). Location: A Neglected Determinant of Firm Growth. Review of World Economics / Weltwirtschaftliches Archiv, 143(1), 79-107.

Audretsch, D.B., Klomp, L., Santarelli, E., \& Thurik, A. R. (2004). Gibrat's Law: Are the Services Different? Review of Industrial Organization, 24(3), 301-324.

Baldwin, J. R., Brown, W. M., \& Rigby, D. L. (2010). Agglomeration economies: microdata panel estimates from canadian manufacturing. Journal of Regional Science, 50(5), 915-934.

Baum, J. R., Locke, E. A., \& Smith, K. G. (2001). A Multidimensional Model of Venture Growth. Academy of Management Journal, 44(2), 292-303.

Beaudry, C., \& Swann, G. M. P. (2009). Firm growth in industrial clusters of the United Kingdom. Small Business Economics, 32(4), 409-424.

Belleau-Arsenault, C., \& Dubé, J. (2019). Quels impacts de l'aide financière Sur la performance des établissements manufacturiers En 
régions non-métropolitaines? Une réponse par méthode d'appariement spatial. Revue Canadienne Des Sciences Régionales, 42, 13.

Bonča, P. D., Tajnikar, M., Ponikvar, N., \& Mörec, B. (2018). Firm growth types and key macroeconomic aggregates through the economic cycle. Journal of Business Economics and Management, 19(1), 138-153.

Borra, C., \& Gómez-García, F. (2016). Wellbeing at Work and the Great Recession: The Effect of Others' Unemployment. Journal of Happiness Studies, 17(5), 1939-1962.

Bricongne, J.-C., Fontagné, L., Gaulier, G., Taglioni, D., \& Vicard, V. (2012). Firms and the global crisis: French exports in the turmoil. Symposium on the Global Dimensions of the Financial Crisis, 87(1), 134-146.

Brown, W. M., \& Rigby, D. L. (2013). Productivité urbaine - qui profite des économies d'agglomération? Statistique Canada, Division de l'analyse économique.

Brunelle, C., \& Dubé, J. (2018). De l'importance de la proximité dans la capacité de résister aux chocs exogènes. Une analyse de survie d'entreprise durant la Grande Récession dans la région périphérique du Bas-Saint-Laurent, Canada. Revue d'Économie Régionale \& Urbaine, Décembre (5-6), 1155-1185.

Brunelle, C., \& Shearmur, R. (2007). L'étalonnage du développement économique régional indicateurs et mesures. Institut national de la recherche scientifique, Urbanisation, culture et société.

Burger, A., Damijan, J. P., Kostevc, Č., \& Rojec, M. (2017). Determinants of firm performance and growth during economic recession: The case of Central and Eastern European countries. Economic Systems, 41(4), 569-590.

Burke, A., Millán, J. M., Román, C., \& van Stel, A. (2018). Exploring the impact of different types of prior entrepreneurial experience on employer firm performance. Journal of Business Research, 90, 107-122.

Cainelli, G., \& Ganau, R. (2018). Distance-based agglomeration externalities and neighbouring firms' characteristics. Regional Studies, 52(7), 922-933.

Cainelli, G., Ganau, R., \& lacobucci, D. (2016). Do Geographic Concentration and Vertically Related Variety Foster Firm Productivity? Micro-Evidence from Italy: Spatial Agglomeration and Firm Productivity. Growth and Change, 47(2), 197-217.

Cissé, I., Dubé, J., \& Brunelle, C. (2020). New business location: how local characteristics influence individual location decision? The Annals of Regional Science, 64(1), 185-214.

Cliff, J. E. (1998). Does one size fit all? exploring the relationship between attitudes towards growth, gender, and business size. Journal of Business Venturing, 13(6), 523-542.

Coad, A. (2007). Firm Growth: A Survey. Université Panthéon-Sorbonne (Paris 1), Centre d'Économie de la Sorbonne.

Coad, A. (2009). The Growth of Firms: A Survey of Theories and Empirical Evidence. Edward Elgar Publishing.

Coad, A., \& Broekel, T. (2012). Firm growth and productivity growth: evidence from a panel VAR. Applied Economics, 44(10), 1251-1269.

Cowling, M., Liu, W., Ledger, A., \& Zhang, N. (2015). What really happens to small and medium-sized enterprises in a global economic recession? UK evidence on sales and job dynamics. International Small Business Journal: Researching Entrepreneurship, 33(5), 488-513.

Criscuolo, C., Gal, P. N., \& Menon, C. (2014). The Dynamics of Employment Growth: New Evidence from 18 Countries. OECD Science, Technology and Industry Policy Papers, 14.
Daunfeldt, S.-O., Elert, N., \& Johansson, D. (2014). The Economic Contribution of High-Growth Firms: Do Policy Implications Depend on the Choice of Growth Indicator? Journal of Industry, Competition and Trade, 14(3), 337-365.

de Groot, H. L. F., Poot, J., \& Smit, M. J. (2016). Which agglomeration externalities matter most and why? Journal of Economic Surveys, 30(4), 756-782.

De Vaan, M., Boschma, R., \& Frenken, K. (2013). Clustering and firm performance in project-based industries: the case of the global video game industry, 1972-2007. Journal of Economic Geography, 13(6), 965-991.

Delmar, F., Davidsson, P., \& Gartner, W. B. (2003). Arriving at the high-growth firm. Journal of Business Venturing, 18(2), 189-216.

Delmar, Frédéric. (1996). Entrepreneurial behavior and business performance. EFI.

Delmar, Frédéric, Davidsson, P., \& Gartner, W. B. (2003). Arriving at the high-growth firm. Journal of Business Venturing, 18(2), 189-216.

Diao, M. (2015). Selectivity, spatial autocorrelation and the valuation of transit accessibility. Urban Studies, 52(1), 159-177.

Dixon, J., \& Rollin, A.-M. (2012). Dynamique des entreprises taux de croissance de l'emploi dans les petites et grandes entreprises au Canada. Statistique Canada, Division de l'analyse économique.

Drinkwater, S., Lashley, J., \& Robinson, C. (2018). Barriers to enterprise development in the Caribbean. Entrepreneurship \& Regional Development, 30(9-10), 942-963.

Dubé, J., \& Brunelle, C. (2014). Dots to dots: a general methodology to build local indicators using spatial micro-data. The Annals of Regional Science, 53(1), 245-272.

Dubé, J., Brunelle, C., \& Legros, D. (2016). Location Theories and Business Location Decision: A Micro-Spatial Investigation in Canada. The Review of Regional Studies, 46(2), 143.

Dubé, J., \& Legros, D. (2014). Économétrie spatiale appliquée des microdonnées. ISTE editions.

Elhorst, J. P. (2010). Applied Spatial Econometrics: Raising the Bar. Spatial Economic Analysis, 5(1), 9-28.

Fingleton, B., Camargo Igliori, D., \& Moore, B. (2004). Employment Growth of Small High-technology Firms and the Role of Horizontal Clustering: Evidence from Computing Services and R\&D in Great Britain, 1991-2000. Urban Studies, 41(4), 773-799.

Fort, T. C., Haltiwanger, J., Jarmin, R. S., \& Miranda, J. (2013). How Firms Respond to Business Cycles: The Role of Firm Age and Firm Size. IMF Economic Review, 61(3), 520-559.

Frey, W. H., \& Johnson, K. M. (1996). Concentrated Immigration, Restructuring and the Selective Deconcentration of the U.S. Population (No. 96-371; p. 33). PSC Research Report.

Fuguitt, G. V., \& Beale, C. L. (1996). Recent Trends in Nonmetropolitan Migration: Toward a New Turnaround? Growth and Change, 27(2), 156-174.

Garnsey, E., Stam, E., \& Heffernan, P. (2006). New Firm Growth: Exploring Processes and Paths. Industry \& Innovation, 13(1), 1-20.

Garsaa, A., \& Levratto, N. (2017). Are disparities in employment growth rates explained by individual or local characteristics? A multilevel analysis on the French employment areas. Region Et Developpement, 45, 31-58.

Giunta, A., Nifo, A., \& Scalera, D. (2012). Subcontracting in Italian Industry: Labour Division, Firm Growth and the North-South Divide. Regional Studies, 46(8), 1067-1083. 
Glaeser, E. L., Kallal, H. D., Scheinkman, J. A., \& Shleifer, A. (1992). Growth in cities. Journal of Political Economy, 100(6), 1126-1152.

Goedhuys, M., \& Sleuwaegen, L. (2016). High-growth versus declining firms: the differential impact of human capital and R\&D. Applied Economics Letters, 23(5), 369-372.

Gupta, P., Guha, S., \& Krishnaswami, S. (2013). Firm growth and its determinants. Journal of Innovation and Entrepreneurship, 2(1), 15.

Haltiwanger, J., Jarmin, R. S., \& Miranda, J. (2013). Who Creates Jobs? Small versus Large versus Young. Review of Economics and Statistics, 95(2), 347-361.

Heckman, J. J. (1979). Sample Selection Bias as a Specification Error. Econometrica, 47(1), 153.

Hoogstra, G. J., \& van Dijk, J. (2004). Explaining Firm Employment Growth: Does Location Matter? Small Business Economics, 22(3/4), 179-192.

ISQ (2019). Profils statistiques de la Région du Bas Saint Laurent. URL: http://www.stat.gouv.qc.ca/statistiques/profils/region_01/ region_01_00.htm

Jovanovic, B. (1982). Selection and the Evolution of Industry. Econometrica, 50(3), 649 .

Kauppila, O.-P. (2015). Alliance Management Capability and Firm Performance: Using Resource-based Theory to Look Inside the Process Black Box. Long Range Planning, 48(3), 151-167.

Kim, W. C., Anselin, L., \& Phipps, T. T. (2003). Measuring the benefits of air quality improvement: a spatial hedonic approach. Journal of Environmental Economics and Management, 45(1), 24-39.

Kiviluoto, N. (2013). Growth as evidence of firm success: myth or reality? Entrepreneurship \& Regional Development, 25(7-8), 569-586.

Lesage, J. P., \& Fischer, M. M. (2008). Spatial Growth Regressions: Model Specification, Estimation and Interpretation. Spatial Economic Analysis, 3(3), 275-304.

LeSage, J. P., \& Pace, R. K. (2009). Introduction to spatial econometrics. CRC Press; Ariane. Accès via Taylor \& Francis eBooks

LeSage, J. P., \& Pace, R. K. (2014). Interpreting Spatial Econometric Models. In M. M. Fischer \& P. Nijkamp (Eds.), Handbook of Regional Science (pp. 1535-1552). Springer Berlin Heidelberg.

Lyee, N., \& Cowling, M. (2015). Do Rural Firms Perceive Different Problems? Geography, Sorting, and Barriers to Growth in UK SMEs. Environment and Planning C: Government and Policy, 33(1), 25-42.

Macpherson, A., \& Holt, R. (2007). Knowledge, learning and small firm growth: A systematic review of the evidence. Research Policy, 36(2), 172-192.

Mameli, F., Faggian, A., \& Mccann, P. (2014). Estimation of Local Employment Growth: Do Sectoral Aggregation and Industry Definition Matter? Regional Studies, 48(11), 1813-1828.

Manzocchi, S., Quintieri, B., \& Santoni, G. (2017). Local manufacturing productivity markers: an empirical study of the Italian counties. The Annals of Regional Science, 59(1), 255-279.

Nelson, L., \& Nelson, P. B. (2011). The global rural: Gentrification and linked migration in the rural USA. Progress in Human Geography, 35(4), 441-459.

Nicholson, N. (1998). Personality and entrepreneurial leadership: European Management Journal, 16(5), 529-539.

Partridge, M. D., Rickman, D. S., Ali, K., \& Olfert, M. R. (2010). Recent spatial growth dynamics in wages and housing costs: Proximity to urban production externalities and consumer amenities. Regional Science and Urban Economics, 40(6), 440-452.
Peric, M., \& Vitezic, V. (2016). Impact of global economic crisis on firm growth. Small Business Economics, 46(1), 1-12.

Piacentino, D., Bono, F., Cracolici, M. F., \& Giuliani, D. (2017). A spatial analysis of new business formation: Replicative vs innovative behaviour. Spatial Statistics, 21, 390-405.

Richard, P. J., Devinney, T. M., Yip, G. S., \& Johnson, G. (2009). Measuring Organizational Performance: Towards Methodological Best Practice. Journal of Management, 35(3), 718-804.

Rosenthal, S. S., \& Strange, W. C. (2004). Evidence on the nature and sources of agglomeration economies. Handbook of Regional and Urban Economics, 4, 2119-2171.

Rupasingha, A., Liu, Y., \& Partridge, M. (2015). Rural Bound: Determinants of Metro to Non-Metro Migration in the United States. American Journal of Agricultural Economics, 97(3), 680-700.

Santarelli, E., \& Vivarelli, M. (2007). Entrepreneurship and the process of firms' entry, survival and growth. Industrial and Corporate Change, 16(3), 455-488.

Serrasqueiro, Z., Nunes, P. M., Leitao, J., \& Armada, M. (2010). Are there non-linearities between SME growth and its determinants? A quantile approach. Industrial and Corporate Change, 19(4), 1071-1108.

Statistique Canada. (2015). Census metropolitan area (CMA) and census agglomeration (CA) - Census Dictionary. https://www12. statcan.gc.ca/census-recensement/2011/ref/dict/geo009-eng.cfm

Steimetz, S. S. C. (2010). Spatial Multipliers in Hedonic Analysis: A Comment on "Spatial Hedonic Models of Airport Noise, Proximity, and Housing Prices." Journal of Regional Science, 50(5), 995-998.

Tang, A. (2015). Does Gibrat's law hold for Swedish energy firms? Empirical Economics, 49(2), 659-674.

van Oort, F. G., Burger, M. J., Knoben, J., \& Raspe, O. (2012). Multilevel approaches and the firm-agglomeration ambiguity in economic growth studies: multilevel approaches and the firm-agglomeration ambiguity. Journal of Economic Surveys, 26(3), 468-491.

Wall, T. D., Michie, J., Patterson, M., Wood, S. J., Sheehan, M., Clegg, C. W., \& West, M. (2004). On the validity of subjective measures of company performance. Personnel Psychology, 57(1), 95-118.

Wang, C. C. (2015). Geography of Knowledge Sourcing, Search Breadth and Depth Patterns, and Innovative Performance: A Firm Heterogeneity Perspective. Environment and Planning A, 47(3), 744761.

Wiklund, J., Patzelt, H., \& Shepherd, D. A. (2009). Building an integrative model of small business growth. Small Business Economics, 32(4), 351-374.

Zhou, H., Huang, L., \& Kuo, T.-K. (2018). Determinants of Small Firm Growth: An Exhaustive Analysis Using Conceptual and Statistical Approaches. International Review of Entrepreneurship, 16(4), 525564. 
Annexe 1. Formules de calcul de ces indices de caractéristiques locales

\begin{tabular}{|c|c|}
\hline $\begin{array}{l}\boldsymbol{i} \text { : nouvel établissement }(\mathrm{i}=1,2, \ldots, \mathrm{N}) \\
\boldsymbol{s}: \text { secteur économique }(\mathrm{s}=1,2, . ., \mathrm{S}) \\
\boldsymbol{r}: \text { rayon de } 1250 \text { mètres } \\
\boldsymbol{R} \text { : L'ensemble de la région du BSL }\end{array}$ & $\begin{array}{l}\text { e oirs ou } \boldsymbol{E}_{\text {oirs }} \text { nombre d'employé ou } \\
\text { d'établissements un an ou deux avant la période } \\
\text { initiale, autour ( } \boldsymbol{r}=1250 \text { mètres }) \text { de } \boldsymbol{i} \text { selon le secteur } \\
\text { économique } \boldsymbol{s}\end{array}$ \\
\hline $\mathbf{Q L}_{\mathbf{i s}}=\frac{p_{i s}}{p_{s}}=\frac{e_{0 i r s} / \sum_{s=1}^{S} e_{0 i r s}}{\sum_{r=1}^{R} e_{0 i r s} / \sum_{s=1}^{S} \sum_{r=1}^{R} e_{0 i r s}}$ & \\
\hline $\boldsymbol{H}_{\boldsymbol{i}}=1-\sum_{\mathrm{s}=1}^{\mathrm{s}} \mathrm{p}_{\mathrm{is}}^{2}$ & \\
\hline$C_{i}=\frac{E_{0 i r s} / e_{0 i r s}}{\sum_{r=1}^{R} E_{0 i r s} / \sum_{r=1}^{R} e_{0 i r s}}$ & \\
\hline $\boldsymbol{R} \boldsymbol{V}_{\boldsymbol{i}}=\sum_{\mathrm{s}=1}^{\mathrm{S}} \mathrm{P}_{\mathrm{s}} \cdot \mathrm{G}_{\mathrm{s}}$ & $\begin{array}{l}\boldsymbol{G}_{\boldsymbol{s}}=\sum_{\mathrm{ss}}^{\mathrm{SS}} \frac{\mathrm{p}_{\mathrm{iss}}}{\mathrm{p}_{\mathrm{s}}} \log \left(\frac{1}{p_{\text {iss }} / p_{\text {is }}}\right) ; s s \in s \\
\boldsymbol{p}_{\text {iss }}: \text { part du sous secteur }(\mathrm{ss})\end{array}$ \\
\hline $\boldsymbol{P M}_{\boldsymbol{i}}=\sum_{j=1}^{J} \frac{M_{j}}{d_{i j}} \quad \forall i \neq j$ & $\begin{array}{l}\mathbf{M}_{\mathbf{j}} \text { : emploi dans les } \boldsymbol{j} \text { autres établissements } \\
\mathbf{d}_{\mathbf{i j}}: \text { distance entre l'établissement } \boldsymbol{i} \text { et } \boldsymbol{j}\end{array}$ \\
\hline
\end{tabular}




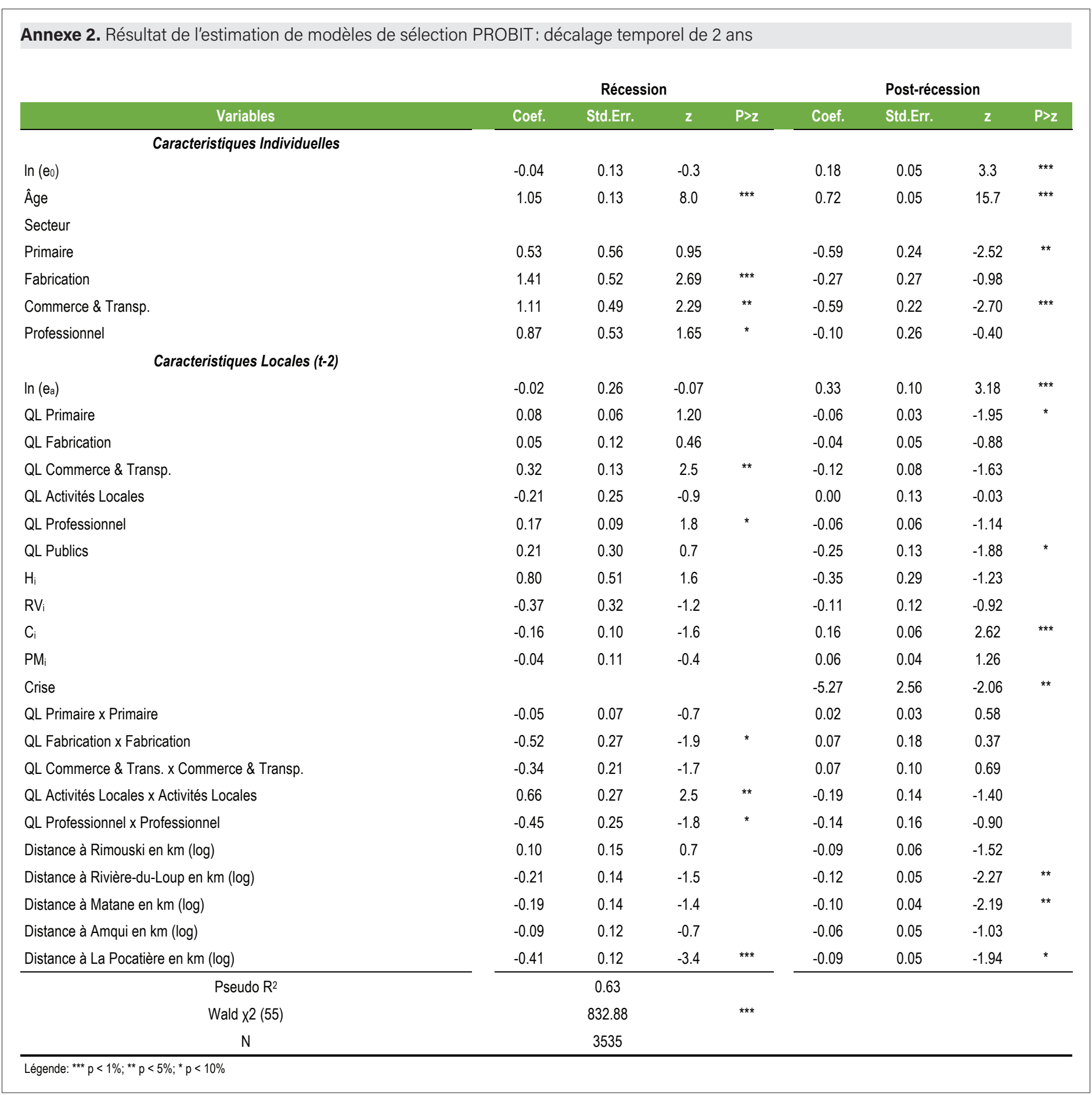


Annexe 3. Impact des changements des variables explicatives, les effets: décalage temporel de 2 ans

5 plus proches observations

\begin{tabular}{|c|c|c|c|c|c|c|c|c|c|c|c|c|}
\hline \multirow{3}{*}{ Variables } & \multirow{2}{*}{\multicolumn{6}{|c|}{ Récession }} & \multirow{2}{*}{\multicolumn{6}{|c|}{ Post récession }} \\
\hline & & & & & & & & & & & & \\
\hline & $\begin{array}{l}\text { Effects } \\
\text { directs }\end{array}$ & Sign. & $\begin{array}{l}\text { Effects } \\
\text { indirects }\end{array}$ & Sign. & $\begin{array}{l}\text { Effects } \\
\text { totaux }\end{array}$ & Sign. & $\begin{array}{l}\text { Effects } \\
\text { directs }\end{array}$ & Sign. & $\begin{array}{l}\text { Effects } \\
\text { indirects }\end{array}$ & Sign. & $\begin{array}{l}\text { Effects } \\
\text { totaux }\end{array}$ & Sign. \\
\hline \multicolumn{13}{|l|}{ Caractéristiques Individuelles } \\
\hline $\ln \left(e_{0}\right)$ & -0.0216 & $* * *$ & 0.0001 & & -0.0215 & * & -0.0007 & & 0.0019 & & 0.0012 & \\
\hline Âge & -0.0468 & $* * *$ & -0.0006 & & -0.0473 & $* * *$ & 0.0448 & $* * *$ & -0.0002 & & 0.0446 & $* * *$ \\
\hline \multicolumn{13}{|l|}{ Secteurs } \\
\hline Primaire & 0.0371 & * & -0.0105 & & 0.0266 & & -0.0177 & ** & 0.0138 & & -0.0039 & \\
\hline Fabrication & 0.0434 & * & -0.0335 & & 0.0099 & & 0.0000 & & -0.0106 & & -0.0106 & \\
\hline Commerce \& Transp. & 0.0425 & ** & -0.0146 & & 0.0279 & & -0.0180 & ** & -0.0013 & & -0.0193 & \\
\hline Professionnel & 0.0141 & & -0.0381 & & -0.0241 & & -0.0033 & & 0.0064 & & 0.0031 & \\
\hline \multicolumn{13}{|l|}{ Caracteristiques Locales (t-2) } \\
\hline $\ln \left(e_{a}\right)$ & -0.0076 & & 0.0000 & & -0.0076 & & 0.0135 & $* \star *$ & -0.0001 & $* * *$ & 0.0134 & $* * *$ \\
\hline QL Primaire & 0.0028 & & 0.0000 & & 0.0028 & & -0.0029 & $* * *$ & 0.0000 & ** & -0.0029 & ** \\
\hline QL Fabrication & -0.0025 & & 0.0000 & & -0.0025 & & -0.0016 & & 0.0000 & & -0.0016 & \\
\hline QL Commerce \& Transp. & 0.0121 & * & 0.0000 & * & 0.0121 & * & -0.0033 & & 0.0000 & & -0.0033 & \\
\hline QL Activités Locales & -0.0005 & & 0.0000 & & -0.0005 & & -0.0018 & & 0.0000 & & -0.0018 & \\
\hline QL Professionnel & -0.0030 & & 0.0000 & & -0.0030 & & -0.0022 & & 0.0000 & & -0.0021 & \\
\hline QL Publics & 0.0049 & & 0.0000 & & 0.0049 & & -0.0093 & * & 0.0000 & & -0.0093 & \\
\hline $\mathrm{H}_{\mathrm{i}}$ & -0.0178 & & 0.0001 & & -0.0178 & & -0.0036 & & 0.0000 & & -0.0036 & \\
\hline $\mathrm{RV}_{\mathrm{i}}$ & 0.0087 & & 0.0000 & & 0.0087 & & -0.0017 & & 0.0000 & & -0.0017 & \\
\hline $\mathrm{Ci}_{\mathrm{i}}$ & -0.0118 & $* * *$ & 0.0000 & $* * *$ & -0.0118 & $* * *$ & 0.0079 & $* * *$ & 0.0000 & $* * *$ & 0.0079 & $* * *$ \\
\hline $\mathrm{PM}_{\mathrm{i}}$ & -0.0047 & & 0.0000 & & -0.0047 & & 0.0014 & & 0.0000 & & 0.0014 & \\
\hline QL Primaire $x$ Primaire & -0.0012 & & 0.0000 & & -0.0012 & & 0.0011 & & 0.0000 & & 0.0011 & \\
\hline QL Fabrication $x$ Fabrication & -0.0090 & & 0.0000 & & -0.0090 & & 0.0066 & & 0.0000 & & 0.0066 & \\
\hline QL Commerce \& Trans. x Commerce \& Transp. & 0.0126 & * & 0.0000 & & 0.0125 & & -0.0033 & & 0.0000 & & -0.0033 & \\
\hline QL Activités Locales x Activités Locales & 0.0095 & & 0.0000 & & 0.0095 & & -0.0053 & & 0.0000 & & -0.0052 & \\
\hline QL Professionnel x Professionnel & -0.0028 & & 0.0000 & & -0.0028 & & -0.0051 & & 0.0000 & & -0.0051 & \\
\hline Distance à Rimouski en km (log) & 0.0026 & & 0.0000 & & 0.0026 & & -0.0113 & $* * *$ & 0.0000 & *** & -0.0113 & *** \\
\hline Distance à Rivière-du-Loup en km (log) & -0.0009 & & 0.0000 & & -0.0009 & & -0.0081 & *** & 0.0000 & $* * *$ & -0.0081 & *** \\
\hline Distance à Matane en km (log) & -0.0058 & & 0.0000 & & -0.0057 & & -0.0035 & * & 0.0000 & & -0.0035 & \\
\hline Distance à Amqui en km (log) & 0.0020 & & 0.0000 & & 0.0020 & & -0.0063 & $* * *$ & 0.0000 & ** & -0.0063 & ** \\
\hline Distance à La Pocatière en km (log) & -0.0016 & & 0.0000 & & -0.0016 & & -0.0049 & ** & 0.0000 & * & -0.0049 & * \\
\hline Crise & & & & & & & 0.2335 & $* * *$ & -0.0009 & $* *$ & 0.2326 & ** \\
\hline
\end{tabular}

Légende: ${ }^{* * *} p<1 \% ;{ }^{* *} p<5 \% ;{ }^{*} p<10 \%$ 
Annexe 3. Impact des changements des variables explicatives, les effets: décalage temporel de 2 ans (suite)

20 plus proches observations

\begin{tabular}{|c|c|c|c|c|c|c|c|c|c|c|c|c|}
\hline \multirow[b]{3}{*}{ Variables } & \multicolumn{12}{|c|}{20 plus proches observations } \\
\hline & \multicolumn{6}{|c|}{ Récession } & \multicolumn{6}{|c|}{ Post récession } \\
\hline & $\begin{array}{l}\text { Effects } \\
\text { directs }\end{array}$ & Sign. & $\begin{array}{l}\text { Effects } \\
\text { indirects }\end{array}$ & Sign. & $\begin{array}{l}\text { Effects } \\
\text { totaux }\end{array}$ & Sign. & $\begin{array}{l}\text { Effects } \\
\text { directs }\end{array}$ & Sign. & $\begin{array}{l}\text { Effects } \\
\text { indirects }\end{array}$ & Sign. & $\begin{array}{l}\text { Effects } \\
\text { totaux }\end{array}$ & Sign. \\
\hline \multicolumn{13}{|l|}{ Caractéristiques Individuelles } \\
\hline $\ln \left(\mathrm{e}_{0}\right)$ & -0.0193 & $* * *$ & -0.0062 & & -0.0255 & & -0.0010 & & 0.0066 & & 0.0056 & \\
\hline Âge & -0.0470 & $* * *$ & 0.0096 & & -0.0374 & $* * *$ & 0.0454 & *** & -0.0075 & * & 0.0379 & $* * *$ \\
\hline \multicolumn{13}{|l|}{ Secteurs } \\
\hline Primaire & 0.0308 & * & 0.0052 & & 0.0360 & & -0.0178 & * & 0.0158 & & -0.0020 & \\
\hline Fabrication & 0.0439 & * & -0.0180 & & 0.0259 & & -0.0004 & & -0.0138 & & -0.0142 & \\
\hline Commerce \& Transp. & 0.0400 & * & -0.1130 & ** & -0.0729 & & -0.0190 & * & 0.0047 & & -0.0143 & \\
\hline Professionnel & 0.0147 & & -0.0131 & & 0.0016 & & -0.0036 & & -0.0010 & & -0.0046 & \\
\hline \multicolumn{13}{|l|}{ Caracteristiques Locales (t-2) } \\
\hline $\ln \left(e_{a}\right)$ & -0.0042 & & 0.0004 & & -0.0038 & & 0.0133 & $* * *$ & -0.0012 & $* * *$ & 0.0120 & $* * *$ \\
\hline QL Primaire & 0.0022 & & -0.0002 & & 0.0020 & & -0.0030 & $* *$ & 0.0003 & ** & -0.0027 & $* *$ \\
\hline QL Fabrication & -0.0026 & & 0.0002 & & -0.0023 & & -0.0021 & & 0.0002 & & -0.0019 & \\
\hline QL Commerce \& Transp. & 0.0139 & ** & -0.0013 & ** & 0.0126 & $* *$ & -0.0041 & & 0.0004 & & -0.0037 & \\
\hline QL Activités Locales & 0.0008 & & -0.0001 & & 0.0007 & & -0.0025 & & 0.0002 & & -0.0023 & \\
\hline QL Professionnel & -0.0038 & & 0.0004 & & -0.0034 & & -0.0020 & & 0.0002 & & -0.0018 & \\
\hline QL Publics & 0.0021 & & -0.0002 & & 0.0019 & & -0.0095 & & 0.0009 & & -0.0086 & \\
\hline $\mathrm{H}_{\mathrm{i}}$ & -0.0270 & & 0.0025 & & -0.0245 & & -0.0032 & & 0.0003 & & -0.0029 & \\
\hline $\mathrm{RV}_{\mathrm{i}}$ & 0.0114 & & -0.0011 & & 0.0103 & & -0.0008 & & 0.0001 & & -0.0007 & \\
\hline $\mathrm{C}_{\mathrm{i}}$ & -0.0118 & $* * *$ & 0.0011 & $* * *$ & -0.0107 & $* * *$ & 0.0080 & $* * *$ & -0.0007 & $* * *$ & 0.0073 & $* \star *$ \\
\hline $\mathrm{PMi}_{\mathrm{i}}$ & -0.0002 & & 0.0000 & & -0.0002 & & 0.0005 & & -0.0001 & & 0.0005 & \\
\hline QL Primaire $x$ Primaire & -0.0007 & & 0.0001 & & -0.0006 & & 0.0012 & & -0.0001 & & 0.0011 & \\
\hline QL Fabrication x Fabrication & -0.0070 & & 0.0007 & & -0.0064 & & 0.0067 & & -0.0006 & & 0.0061 & \\
\hline QL Commerce \& Trans. $x$ Commerce \& Transp. & 0.0147 & ** & -0.0014 & & 0.0134 & & -0.0029 & & 0.0003 & & -0.0026 & \\
\hline QL Activités Locales x Activités Locales & 0.0087 & & -0.0008 & & 0.0079 & & -0.0051 & & 0.0005 & & -0.0046 & \\
\hline QL Professionnel x Professionnel & -0.0042 & & 0.0004 & & -0.0038 & & -0.0043 & & 0.0004 & & -0.0039 & \\
\hline Distance à Rimouski en km (log) & 0.0072 & & -0.0007 & & 0.0065 & & -0.0118 & $* * *$ & 0.0011 & *** & -0.0107 & $* * *$ \\
\hline Distance à Rivière-du-Loup en km (log) & -0.0030 & & 0.0003 & & -0.0027 & & -0.0069 & ** & 0.0006 & ** & -0.0062 & ** \\
\hline Distance à Matane en km (log) & -0.0061 & & 0.0006 & & -0.0055 & & -0.0026 & & 0.0002 & & -0.0024 & \\
\hline Distance à Amqui en km (log) & -0.0026 & & 0.0002 & & -0.0023 & & -0.0056 & * & 0.0005 & * & -0.0050 & * \\
\hline Distance à La Pocatière en km (log) & -0.0023 & & 0.0002 & & -0.0021 & & -0.0048 & * & 0.0004 & * & -0.0043 & * \\
\hline Crise & & & & & & & 0.2314 & * & -0.0216 & * & 0.2098 & * \\
\hline
\end{tabular}

Légende: : ${ }^{* *} p<1 \%$; ** $p<5 \% ;{ }^{*} p<10 \%$ 